\title{
Genomic and transcriptomic hallmarks of poorly differentiated and anaplastic thyroid cancers
}

\author{
Iñigo Landa, ${ }^{1}$ Tihana Ibrahimpasic, ${ }^{2}$ Laura Boucai, ${ }^{3}$ Rileen Sinha, ${ }^{4,5}$ Jeffrey A. Knauf, ${ }^{1,3}$ Ronak H. Shah, ${ }^{1}$ Snjezana Dogan, ${ }^{6}$ \\ Julio C. Ricarte-Filho, ${ }^{1}$ Gnana P. Krishnamoorthy, ${ }^{1}$ Bin Xu, ${ }^{6}$ Nikolaus Schultz, ${ }^{7,8}$ Michael F. Berger, ${ }^{1,6,8}$ Chris Sander, ${ }^{4}$ \\ Barry S. Taylor, ${ }^{1,7}$ Ronald Ghossein, ${ }^{6}$ Ian Ganly, ${ }^{1,2}$ and James A. Fagin ${ }^{1,3}$ \\ ${ }^{1}$ Human Oncology and Pathogenesis Program, ${ }^{2}$ Department of Surgery, ${ }^{3}$ Department of Medicine, and ${ }^{4}$ Computational Biology Program, Memorial Sloan Kettering Cancer Center, New York, New York, USA. \\ ${ }^{5}$ Department of Genetics and Genomic Sciences, Icahn Institute of Cenomics and Multiscale Biology, Icahn School of Medicine at Mount Sinai, New York, New York, USA. ${ }^{6}$ Department of Pathology, \\ ${ }^{7}$ Department of Epidemiology and Biostatistics, and ${ }^{8}$ Marie-Josée and Henry R. Kravis Center for Molecular Oncology, Memorial Sloan Kettering Cancer Center, New York, New York, USA.
}

BACKGROUND. Poorly differentiated thyroid cancer (PDTC) and anaplastic thyroid cancer (ATC) are rare and frequently lethal tumors that so far have not been subjected to comprehensive genetic characterization.

METHODS. We performed next-generation sequencing of 341 cancer genes from 117 patient-derived PDTCs and ATCs and analyzed the transcriptome of a representative subset of $\mathbf{3 7}$ tumors. Results were analyzed in the context of The Cancer Genome Atlas study (TCGA study) of papillary thyroid cancers (PTC).

RESULTS. Compared to PDTCs, ATCs had a greater mutation burden, including a higher frequency of mutations in TP53, TERT promoter, PI3K/AKT/mTOR pathway effectors, SWI/SNF subunits, and histone methyltransferases. BRAF and RAS were the predominant drivers and dictated distinct tropism for nodal versus distant metastases in PDTC. RAS and BRAF sharply distinguished between PDTCs defined by the Turin (PDTC-Turin) versus MSKCC (PDTC-MSK) criteria, respectively. Mutations of EIF1AX, a component of the translational preinitiation complex, were markedly enriched in PDTCs and ATCs and had a striking pattern of co-occurrence with RAS mutations. While TERT promoter mutations were rare and subclonal in PTCs, they were clonal and highly prevalent in advanced cancers. Application of the TCGA-derived BRAF-RAS score (a measure of MAPK transcriptional output) revealed a preserved relationship with BRAF/RAS mutation in PDTCs, whereas ATCs were BRAF-like irrespective of driver mutation.

CONCLUSIONS. These data support a model of tumorigenesis whereby PDTCs and ATCs arise from well-differentiated tumors through the accumulation of key additional genetic abnormalities, many of which have prognostic and possible therapeutic relevance. The widespread genomic disruptions in ATC compared with PDTC underscore their greater virulence and higher mortality.

FUNDING. This work was supported in part by NIH grants CA50706, CA72597, P50-CA72012, P30-CA008748, and 5T32CA160001; the Lefkovsky Family Foundation; the Society of Memorial Sloan Kettering; the Byrne fund; and Cycle for Survival.

\section{Introduction}

A comprehensive investigation of the genomic landscape of papillary thyroid carcinomas (PTC), the most common thyroid malignancy, was recently reported by The Cancer Genome Atlas Network (TCGA Network) (1). These well-differentiated tumors were found to have a low frequency of somatic alterations (2), with the majority harboring mutually exclusive activating mutations in $B R A F(60 \%)$ and RAS-family genes (13\%), as well as fusion oncoproteins, primarily involving receptor tyrosine kinases (RTKs) such as RET, NTRK1 or -3 , and $A L K$. Distinct signaling and transcriptomic consequences were observed between $\mathrm{BRAF}^{\mathrm{V} 600 \mathrm{E}}$-like

Authorship note: I. Landa and T. Ibrahimpasic contributed equally to this work. Conflict of interest: The authors have declared that no conflict of interest exists. Submitted: October 27, 2015; Accepted: January 4, 2016

Reference information: J Clin Invest. 2016;126(3):1052-1066. doi:10.1172/JCI85271. tumors, which showed higher MAPK transcriptional output and lower expression of genes involved in iodine metabolism, and RAS-like tumors, which had lower MAPK signaling and comparatively preserved expression of iodine-related genes.

The TCGA study excluded poorly differentiated thryoid cancers (PDTCs) and anaplastic thyroid cancers (ATCs) from their analysis in order to focus on a homogeneous histological cohort that would provide sufficient power to identify low-frequency genomic events. Although PDTCs and ATCs account for approximately $5 \%-10 \%$ of thyroid cancers, they represent a major clinical challenge. Patients with PDTC and ATC have a mean survival after diagnosis of 3.2 and 0.5 years, respectively, and account for approximately a third of deaths caused by this disease (3). Virtually all cases are refractory to radioiodine therapy, and traditional chemotherapy and radiotherapy are of marginal benefit $(4,5)$. Molecularly targeted approaches are being tested in preclinical 


\section{Table 1. Summary of the clinicopathological features of the $\mathbf{1 1 7}$ advanced thyroid tumors included in the study}

\begin{tabular}{|c|c|c|c|}
\hline & & PDTC & ATC \\
\hline \multicolumn{2}{|l|}{ Number of tumors } & 84 & 33 \\
\hline \multirow{3}{*}{$\begin{array}{l}\text { Sample type } \\
{[\mathrm{N}(\%)]}\end{array}$} & Primary & $64(76.2)$ & 28 (84.9) \\
\hline & Metastasis & $16(19.0)$ & $3(9.0)$ \\
\hline & Recurrence in neck & $4(4.8)$ & $2(6.1)$ \\
\hline \multirow[t]{2}{*}{ Age [years] } & Median & 58 & 66 \\
\hline & Range & $22-87$ & $34-82$ \\
\hline \multirow[t]{2}{*}{$\operatorname{Sex}[N(\%)]$} & Female & $51(60.7)$ & $18(54.5)$ \\
\hline & Female/Male ratio & $1.5: 1$ & $1.2: 1$ \\
\hline \multirow[t]{7}{*}{ Distant metastasis [N (\%)] } & No & $43(51.2)$ & $0(0.0)$ \\
\hline & Yes & $30(35.7)$ & $15(45.5)$ \\
\hline & lung & $16(19.0)$ & $10(30.3)$ \\
\hline & bone & $5(6.0)$ & $2(6.1)$ \\
\hline & lung + bone & $7(8.3)$ & $2(6.1)$ \\
\hline & other & $2(2.4)$ & $1(3.0)$ \\
\hline & unknown & $11(13.1)$ & $18(54.5)$ \\
\hline Survival [N (\%)] & Alive & $57(67.9)$ & $11(33.3)$ \\
\hline \multirow[t]{2}{*}{ Sample preservation [N (\%)] } & Frozen & $17(20.2)$ & $20(60.6)$ \\
\hline & FFPE & $67(79.8)$ & $13(39.4)$ \\
\hline Normal tissue [N (\%)] & Paired normal & $78(92.9)$ & $28(84.8)$ \\
\hline \multirow{6}{*}{$\begin{array}{l}\text { Cytological phenotype } \\
\text { [N (\%)] }\end{array}$} & Papillary & $32(38.1)$ & $7(21.2)$ \\
\hline & Follicular & $18(21.4)$ & $0(0.0)$ \\
\hline & Tall cell & $19(22.6)$ & $5(15.2)$ \\
\hline & Hurthle & $9(10.7)$ & $1(3.0)$ \\
\hline & Mixed/other & $5(6.0)$ & $2(6.1)$ \\
\hline & $\mathrm{N} / \mathrm{A}$ & $1(1.2)$ & $18(54.5)$ \\
\hline \multirow[t]{4}{*}{ PDTC growth pattern [N (\%)] } & Solid & $53(63.1)$ & \\
\hline & Papillary & $23(27.4)$ & \\
\hline & Mixed/other & $7(8.3)$ & \\
\hline & $\mathrm{N} / \mathrm{A}$ & $1(1.2)$ & \\
\hline \multirow[t]{3}{*}{ PDTC definition [N (\%)] } & Turin proposal & $52(61.9)$ & \\
\hline & MSKCC & $31(36.9)$ & \\
\hline & $\mathrm{N} / \mathrm{A}$ & $1(1.2)$ & \\
\hline
\end{tabular}

studies and in early human clinical trials $(6,7)$. These efforts are constrained by the paucity of information on the genomics of these cancers, which have been investigated primarily through Sanger sequencing of a limited set of candidate genes (8-20). The exception to this is a recently reported whole exome sequencing study of ATC (21), which, although informative, may have undercalled significant mutations - particularly those that are subclonal - because of low tumor purity. This is because ATCs, and to a lesser extent PDTCs, pose a particular challenge for genomic studies due to their extensive infiltration by macrophages (22, 23). This cannot be overcome by microdissection of tissue samples because the macrophages form an interconnected network that envelops the individual tumor cells throughout the tumor specimen. To overcome this, we adopted an ultradeep sequencing strategy using the MSK-IMPACT cancer exome panel, a massively parallel exon capture approach that targets all exons and selected introns of 341 genes frequently altered in human cancer (24). We performed this extensive cancer gene exome sequencing as well as expression profiling in the largest series of PDTC and ATC ever investigated, and we identified a wide spectrum of somatic mutations, genetic fusions, and copy number alterations (CNAs) that clearly delineate profound genomic differences between the two advanced forms of the disease. Moreover, when analyzed in the context of the PTC TCGA study (1), this study provides insights into tumor microevolution, suggesting that PDTCs and ATCs evolve from their well-differentiated counterparts.

\section{Results}

\section{Samples, clinical data, and overall approach}

One-hundred and seventeen advanced thyroid tumors, including 84 PDTCs and 33 ATCs, met the sequencing quality standards and are reported in this study. Clinicopathological features are summarized in Table 1 and Supplemental Table 1 (supplemental material available online with this article; doi:10.1172/JCI85271DS1). Median age was 58 and 66 years for PDTC and ATC, respectively. Female/male ratios were 1.5:1 (PDTC) and 1.2:1 (ATC), which are distinct from PTC (2.7:1) (25). Most samples were primary tumors: 92/117 (64/84 PDTC; 28/33 ATC), and the remainder from nodal (6) or distant (19) metastases.

MSK-IMPACT, a next-generation sequencing (NGS) platform for targeted sequencing of 341 cancer genes (24), was performed in all 117 tumors. A subset of 37 (17 PDTCs and 20 ATCs) was also subjected to an mRNA expression array and a CGH platform to validate IMPACT-derived copy number calls, as detailed later. Median tumor purity was $72 \%$ and $42 \%$ for PDTCs and ATCs, respectively, consistent with the known heavy macrophage infiltration in ATCs and highlighting the need for deeper sequence coverage in these tumors (Figure 1B). Average depth of coverage was $584 \times$ for tumors and $236 \times$ for paired normal tissues. Average coverage was $500 \times$ and $765 \times$, for FFPE and frozen tumors, respectively. Coverage for ATCs was $739 x$.

\section{Somatic mutations}

ATCs harbored a higher number of mutations than PDTCs (median \pm interquartile range $[\mathrm{IQR}]: 6 \pm 5$ and $2 \pm 3$, respectively, $P<1 \times 10^{-4}$ ) (Figure 1A). The mutation burden in PDTCs was increased compared with the PTCs from the TCGA study (considering mutations in the 341 gene set only): $2 \pm 3$ and $1 \pm 1$, respectively $\left(P<1 \times 10^{-4}\right)$. These differences remained highly significant after removing tumors with defects in mismatch excision repair (MMR) genes, which showed a disproportionately higher number of mutations. Mutation burden in PDTCs (expressed as number of mutations below, equal, or above the median) was greater in older patients ( 47 vs. 58 vs. 64 years, $\left.P<1 \times 10^{-3}\right)$ and associated with tumor size $(36 \%$ vs. $43 \%$ vs. $71 \%>4 \mathrm{~cm}, P=0.04$ ), presence of distant metastasis ( $8 \%$ vs. $29 \%$ vs. $57 \%, P=2 \times 10^{-3}$ ), and overall survival ( $19 \%$ vs. $25 \%$ vs. $46 \%$, $\operatorname{logrank} P=0.01$ ) (Supplemental Table 2).

Drivers and frequently altered genes. $\mathrm{BRAF}^{\mathrm{V} 600 \mathrm{E}}$ mutations were present in $33 \%$ of PDTCs and $45 \%$ of ATCs, whereas mutations in NRAS, HRAS, or KRAS occurred in $28 \%$ and $24 \%$ of PDTCs and ATCs, respectively, and were mutually exclusive with BRAF and gene fusions (Figure 1, C and D, and Supplemental Tables 3 and 4). There was a dichotomy in the distribution of RAS and BRAF mutations of PDTCs according to their histological features. Ninety- 


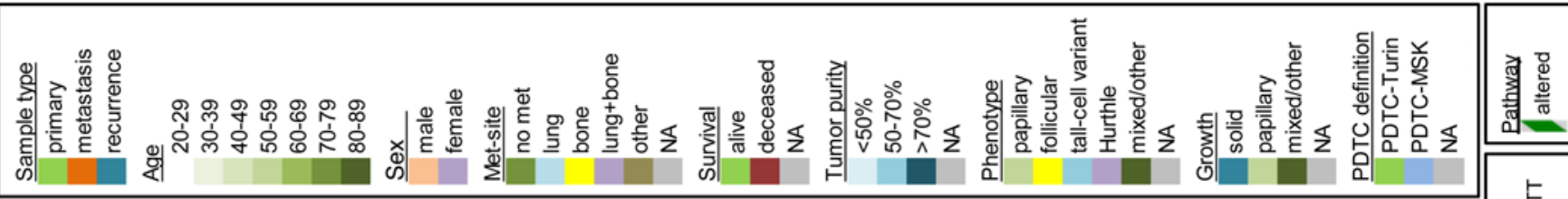
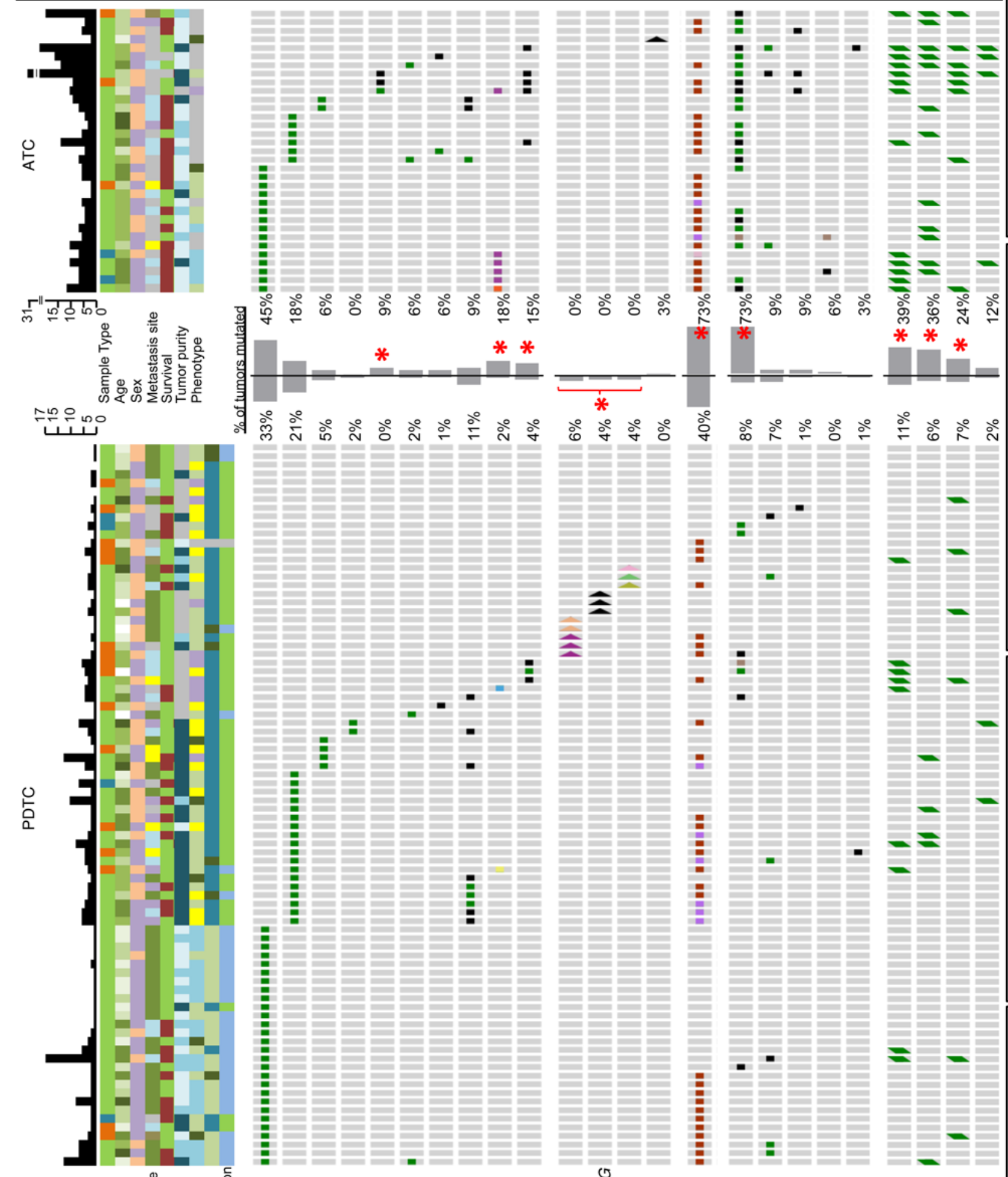

ฟั่
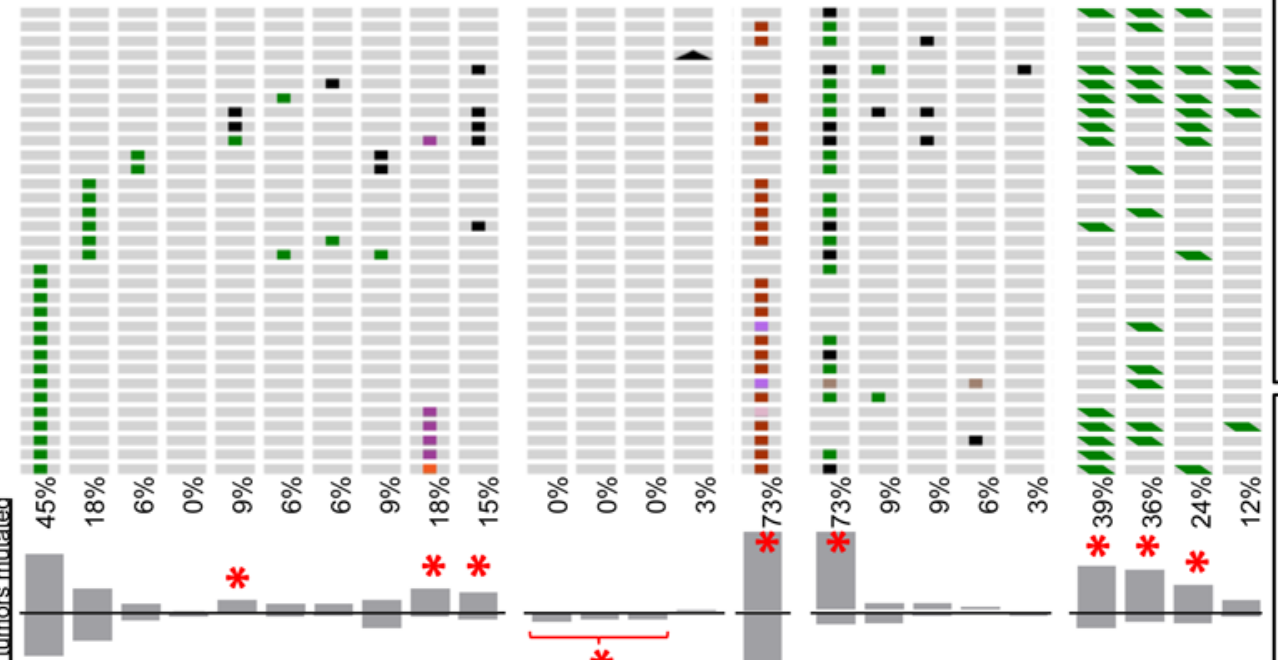

ปู่

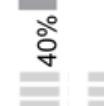

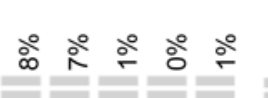

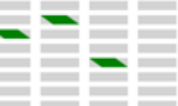

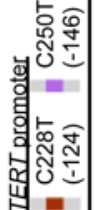

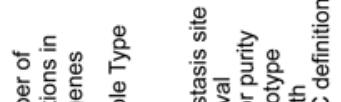

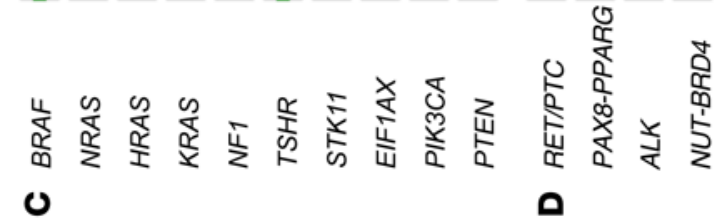
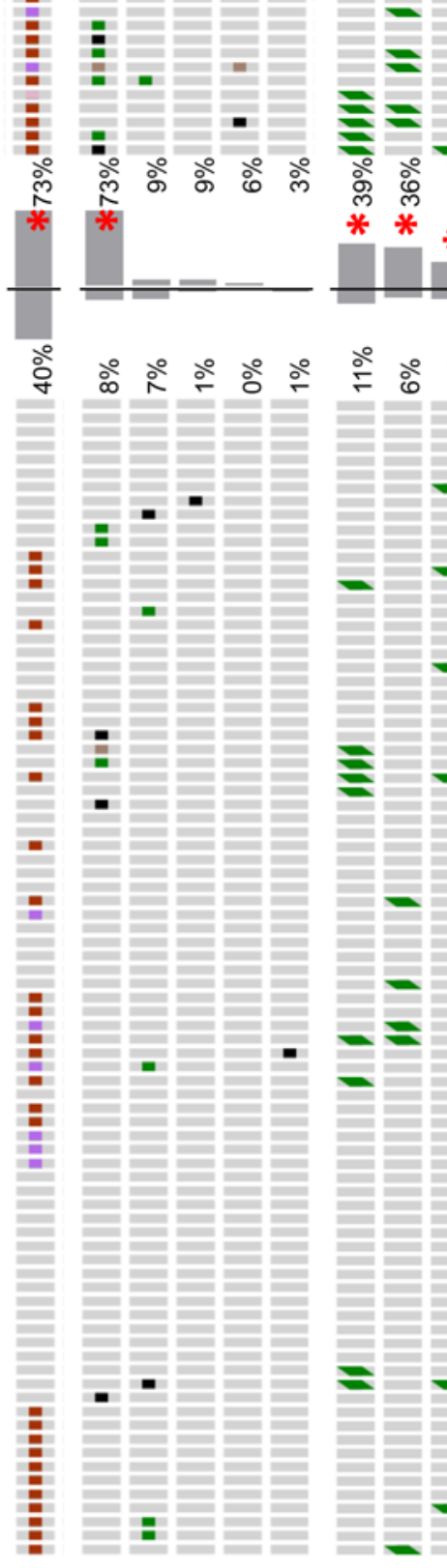

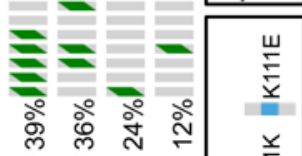

* * *

咅

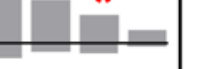

Ф్

ㅇํㅇ ڤे

产新

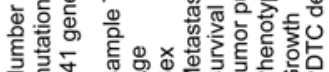

$\varangle \boldsymbol{m}$

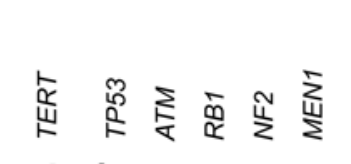

ш 4
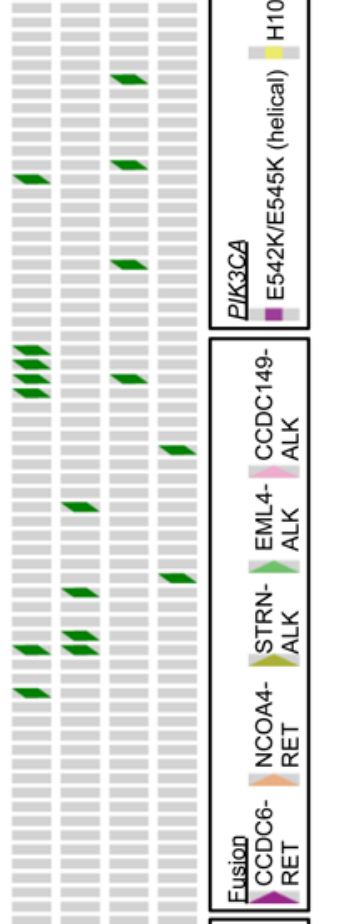

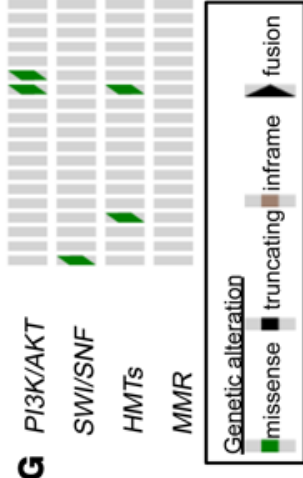


Figure 1. Cancer genome alterations in 84 PDTCs and 33 ATCs. (A) Mutation density across the PDTC and ATC cohorts ( $n=117)$, expressed as number of genetic alterations found in 341 genes present in MSK-IMPACT. (B) Clinicopathological features, including sample type, patients' age (by decade), sex, metastasis site, survival status, tumor purity, cytological phenotype, growth pattern, and PDTC definition (PDTC-Turin vs. PDTCMSK). Color keys are shown in the right outermost panel. (C-G) Oncoprints of PDTCs (left) and ATCs (right). Middle panel shows percentage of tumors altered for each event; ${ }^{*} P<0.05$ between PDTCs vs. ATCs using Fisher's exact test (see Supplemental Table 3 for extended information). Color key for genetic alterations is shown in the bottom panel. Mutations in drivers and other relevant genes (C); fusion events (D); TERT promoter mutations (E); mutations in TP53 and other tumor suppressor genes $(\mathbf{F})$; and alterations in key pathways and functional groups: PI3K/AKT pathway (includes PIK3CA, PTEN, PIK3C2G, PIK3CG, PIK3C3, PIK3R1, PIK3R2, AKT3, TSC1, TSC2, and MTOR), SWI/SNF chromatin remodeling complex (ARID1A, ARID1B, ARID2, ARID5B, SMARCB1, PBRM1, and ATRX), HMTs (KMT2A, KMT2C, KMT2D, and SETD2), and MMR (includes MSH2, MSH6, and MLH1 genes) (C). See Figure 4 for detailed mutational information.

two percent of RAS mutations were found in PDTCs fulfilling the Turin definition of the disease (PDTC-Turin; see Methods, ref. 26). By contrast, $81 \%$ of $B R A F$ mutations were found in PDTCs defined based only on MSKCC criteria (PDTC-MSK): high mitotic rate and necrosis irrespective of growth pattern (ref. 27 and Figure $1, \mathrm{~B}$ and C). BRAF-mutant PDTCs were smaller and had higher frequency of nodal metastases, whereas their RAS-mutant counterparts were larger and had a higher rate of distant metastasis (Supplemental Table 5). BRAF-mutant PDTCs were significantly overrepresented among female patients $(P=0.005)$.

Neurofibromin 1 gene (NF1) mutations were identified in 3 $B R A F / R A S$ WT ATCs. There were also low-frequency mutations in TSHR and STK11 in both PDTCs and ATCs (Figure 1C). Mutations in PIK3CA and PTEN, which encode key effectors of the PI3K/ AKT pathway, were particularly prevalent in ATCs $(18 \%$ and $15 \%$, respectively, Supplemental Table 3) and were overrepresented with respect to PDTCs $\left(P=4 \times 10^{-2}\right.$ and $6 \times 10^{-3}$, respectively). PIK3CA and PTEN showed distinct patterns of co-occurrence in ATCs. All 3 ATCs harboring $N F 1$ mutations also had truncating alterations in PTEN $\left(P=2 \times 10^{-3}\right)$, whereas PIK3CA and BRAF mutations tended to co-occur. All 5 PIK3CA helical domain mutations (E542K or E545K) occurred in ATCs, whereas the single kinase domain mutation (H1047R) was found in a PDTC (Figure 1C).

The EIF1AX-RAS association. Mutations in the eukaryotic translation initiation factor EIF1AX were initially discovered in uveal melanomas (28) and were also reported in 1\% of PTCs (6/402), largely occurring in a mutually exclusive manner with $B R A F$ and RAS (1). By contrast, $11 \%$ of PDTCs and $9 \%$ of ATCs harbored EIF1AX mutations (Figure 1C and Figure 2A), which were strongly associated with $R A S\left(14 / 15, P<1 \times 10^{-4}\right.$, Figure 2B and Table 2). EIF1AX mutations clustered in two regions: the $\mathrm{N}$-terminal domain, as also observed in uveal melanomas (28) and other tumors (Figure 2A), or at a unique splice acceptor site between exons 5 and 6 (p.A113splice), which was the most prevalent abnormality and which has not been reported in other tumor types. The C-terminal p.A113 splice mutation predicts for alternative usage of a cryptic splice acceptor within exon 6, resulting in a 12-amino acid in-frame deletion. Our analysis of RNASeq data from two cases with this mutation in the PTC-TCGA confirms this prediction (not shown). EIF1AX mutations were associated with larger tumors and predicted for shorter survival in PDTCs (Figure 2C and Supplemental Table 6).

TERT in advanced thyroid cancer. There was a high prevalence of telomerase reverse transcriptase (TERT) promoter mutations in these advanced thyroid tumors, and their presence was associated with aggressive and metastatic phenotypes (Figure 1E). Together, $40 \%$ of PDTCs and $73 \%$ of ATCs harbored TERT promoter mutations (49/117 C228T [c.-124G>A]; 8/117 C250T [c.-146G $>$ A]) as compared with 9\% of PTCs from TCGA (Figure 3, A and B, and Table 3). Whereas TERT promoter mutations were subclonal in the small subset of PTC that harbored them, they were clonal in PDTC and ATCs (Figure 3C). TERT mutations co-occurred with $B R A F / R A S$ mutations in PDTCs and ATCs combined $\left(P=4 \times 10^{-3}\right.$, Figure 3B and Table 3), consistent with the proposed mechanism whereby the TERT mutations generate de novo binding elements for ETS-family transcription factors activated by MAPK signaling, such as GABPA (29). Survival of ATC patients harboring TERT promoter mutations was markedly diminished (732 vs. 147 days, $P=0.03$, Supplemental Table 7), particularly in cancers with coexisting mutations of BRAF or RAS (Figure 3D). TERT-mutated PDTCs developed more distant metastases ( $56 \%$ vs. $20 \%, P=0.01$ ) and had a trend toward greater mortality (Supplemental Table 7). RAS-mutant ATCs with TERT (5/8) and EIF1AX mutations (3/8) did not overlap (odds ratio $=0.01 ; P=0.02$ ), consistent with alternate pathways toward progression to ATC.

TP53 and other tumor-suppressor genes. Inactivation of p53 has been considered as a hallmark of advanced thyroid tumors $(14,30)$. We found that TP53 mutations, although highly prevalent in ATCs, were relatively rare in PDTCs ( $73 \%$ vs. $8 \%, P<1 \times 10^{-4}$, Figure $\left.1 \mathrm{~F}\right)$, which contrasts with previous reports (refs. 11, 31, and Supplemental Table 8) and constitutes a key distinguishing event in the biology of these tumors. ATM, a cell-cycle checkpoint and DNA damage response gene, was mutated in 7\% of PDTCs and 9\% of ATCs. ATM-mutated tumors had a higher mutation burden: median was 5 and 2 in $A T M$-mutant versus WT PDTCs $(P=0.04)$, and 19 and 5.5 in ATC, respectively $\left(P=7 \times 10^{-3}\right)$. A higher mutation burden is consistent with the loss of the canonical function of this checkpoint kinase, which is activated in response to DNA double strand breaks and is required for appropriate DNA repair (32). Infrequent truncating mutations were also found in RB1, NF2, and MEN1.

Novel genes and pathways altered in advanced thyroid tumors. Mutations of genes encoding members of the PI3K/AKT/mTOR pathway were seen more frequently in ATCs than PDTCs ( $39 \%$ vs. $11 \%, P=1$ $\left.\times 10^{-3}\right)$. Besides PIK3CA and PTEN, mutations of PIK3C2G, PIK3CG, PIK3C3, PIK3R1, PIK3R2, AKT3, TSC1, TSC2, and MTOR were also present (Figure 1G, Figure 4, A and E, and Supplemental Figure 1).

Genes encoding components of the SWI/SNF chromatin remodeling complex were mutated in $36 \%$ of ATCs and $6 \%$ of PDTCs $\left(P=1 \times 10^{-4}\right)$. This is the first report of mutations in ARID1A, ARID1B, ARID2, ARID5B, SMARCB1, PBRM1, and ATRX genes in advanced thyroid tumors. Consistent with evidence that disruption of one protein in this complex is typically sufficient to impair function (33), we observed a pattern of mutual exclusivity of mutations in this category of genes (Figure 4B). The single exception was for an ATC with concurrent missense mutations in ARIDIA (minor allele fre- 
A

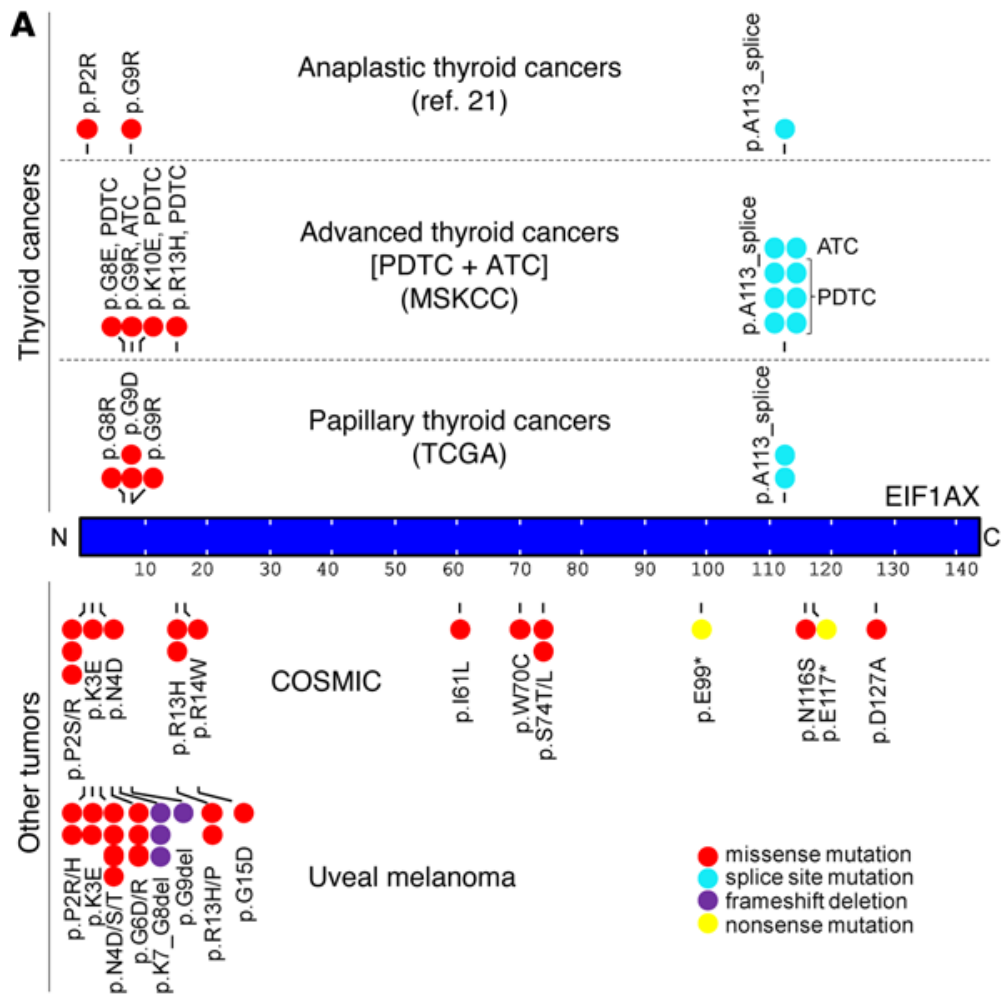

B

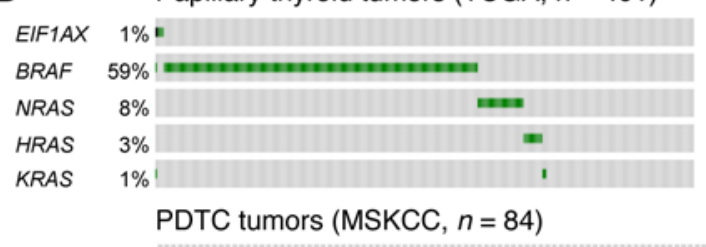

EIF1AX $11 \%$ мими

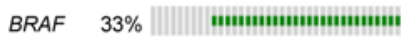

NRAS

HRAS

KRAS

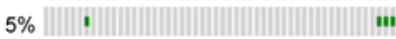

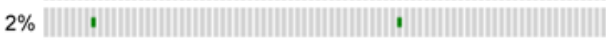

ATC tumors $(n=55)$

$[($ MSKCC,$n=33)+($ ref. $21, n=22)]$

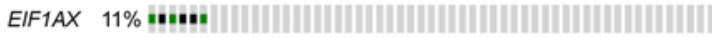

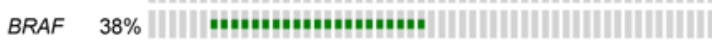

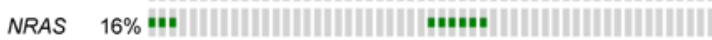

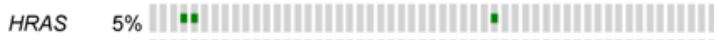

KRAS $4 \%$ IIII| |||||||||||||||||||||||||||||||||||||||||||||||||||||-

Genetic alteration: Missense $\mathbf{m}$ Truncating

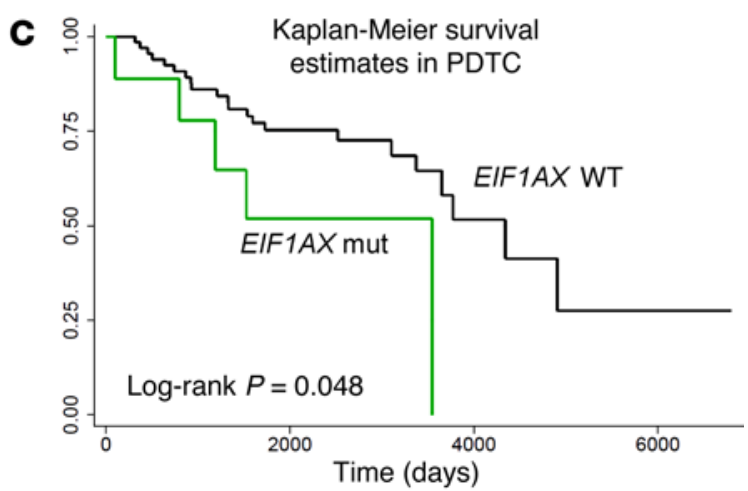

Figure 2. EIF1AX mutations and EIF1AX-RAS co-occurrence in thyroid cancers. (A) Distribution of EIF1AX mutations in thyroid cancers and other tumors (modified from ref. 1). (B) Oncoprints showing the co-occurrence of EIF1AX with RAS mutations in PTC from TCGA (top, $n=401$ ), PDTCs from our study (middle, $n=84$ ), and ATCs from our series and from (21) (bottom, $n=55$ ). (C) Kaplan-Meier graph showing significantly shorter survival in EIF1AX-mutated PDTCs (log-rank $P=0.048)$. See Supplemental Table 6 for detailed clinical correlations.

quency $[\mathrm{MAF}]=5 \%), A R I D 1 B(\mathrm{MAF}=6 \%)$, and a frameshift change in SMARCB1 (MAF $=22 \%$ ), although their frequencies suggest that they are likely subclonal events within a heterogeneous tumor.

Mutations of the histone methyltransferases (HMTs) KMT2A, KMT2C, KMT2D, and SETD2 were found in 24\% of ATCs and 7\% of PDTCs $(P=0.02)$ (Figure $4 C)$. Additional mutations in chromatin remodeling and epigenetic regulators other than SWI/SNF and HMTs were also seen, including frequent alterations affecting the histone acetyltransferase $C R E B B P$ and sporadic inactivating mutations in other epigenetic players such as $E P 300, B C O R$, and BCL6 (Supplemental Figure 1). Mutations in a few epigenetic regulators (ARID1B, KMT2A, and KMT2C) were also identified in $1 \%-2 \%$ of PTCs from the TCGA, but these events are clearly enriched in advanced thyroid tumors.

Alterations in members of the DNA MMR pathway, including $M S H 2, M S H 6$, and $M L H 1$, were found in $12 \%$ of ATCs and $2 \%$ of PDTCs (Figure 4D). MMR mutant tumors showed a "hypermutator phenotype" (as described in ATCs; ref. 21): median mutation number in MMR-mutant vs. WT ATCs was 16.5 and $5\left(P=1 \times 10^{-3}\right)$ and, in PDTCs, 7.5 and $2\left(P=9 \times 10^{-3}\right)$, respectively.
Other genes and functional categories were mutated in a small proportion of PDTCs and ATCs (Supplemental Figure 1 and Supplemental Table 4), i.e., RTKs such as EPHA3 (3 mutations, exclusively in ATCs), EGFR, FLT1 (VEGFR1), FLT4 (VEGFR3), and KDR (VEGFR2), as well as in all four members of the NOTCH family (NOTCH1-4). Finally, there were infrequent

\begin{tabular}{|c|c|c|c|}
\hline & RASWT & $R A S$ mutant & Total \\
\hline EIF1AX WT & $100(80.6)$ & $24(19.4)$ & $124(100.0)$ \\
\hline EIF1AX mutant & $1(6.7)$ & $14(93.3)$ & 15 (100.0) \\
\hline Total & 101 & 38 & 139 \\
\hline
\end{tabular}

Number of samples and percentage (in parentheses) of RAS mutations in EIF1AX WT and mutant samples. The ATC series combines 33 samples from this study and 22 from Kuntsman et al. (21). Statistical analysis show a highly significant co-occurrence of EIFTAX and RAS mutations (odds ratio $=58.3 ; 2$-tailed Fisher's exact test associated $P<0.001$ ). 
A

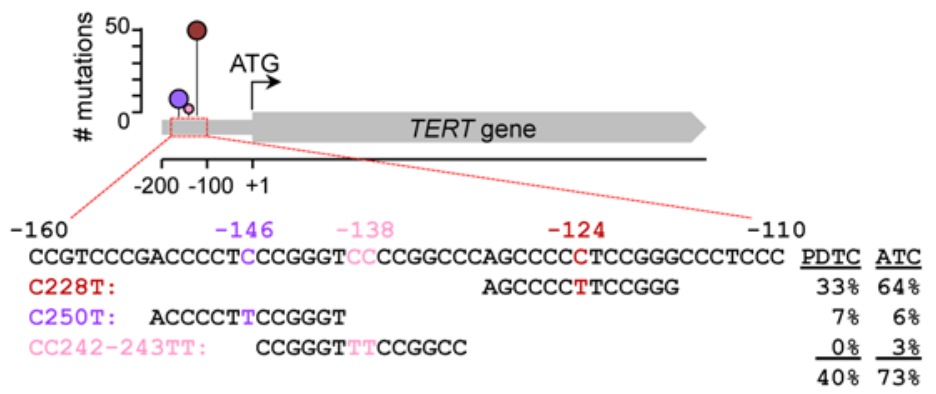

B

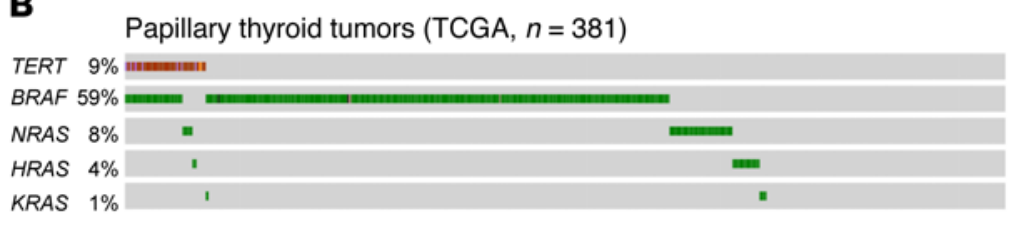

PDTC tumors $(n=84)$

TERT 40\% Hini,

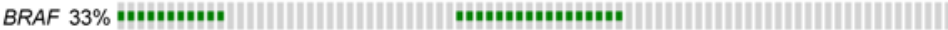

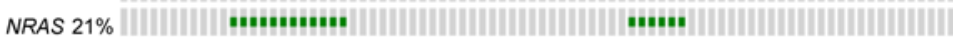
HRAS 5\% IIIIIIIIIIIIIIIIIIIII|,

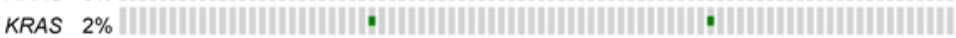

\begin{tabular}{|c|c|c|}
\hline & $\operatorname{rs}(n=$ & \\
\hline TERT 73\% & 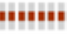 & \\
\hline BRAF $45 \%$ & & ! \\
\hline NRAS $18 \%$ & I."in & II \\
\hline HRAS $6 \%$ & & ". \\
\hline KRAS $\quad 0 \%$ & & \\
\hline
\end{tabular}

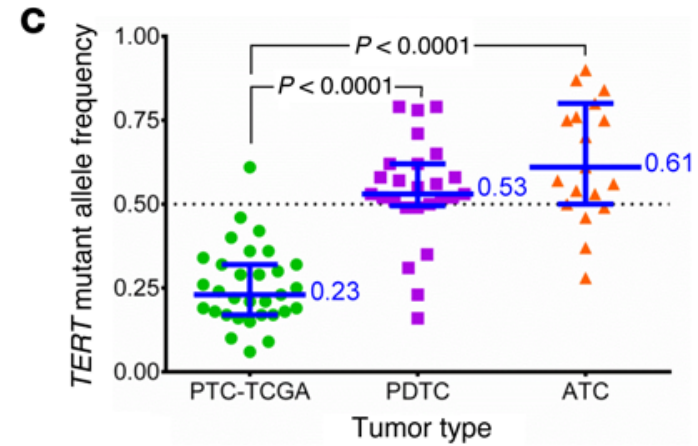

D

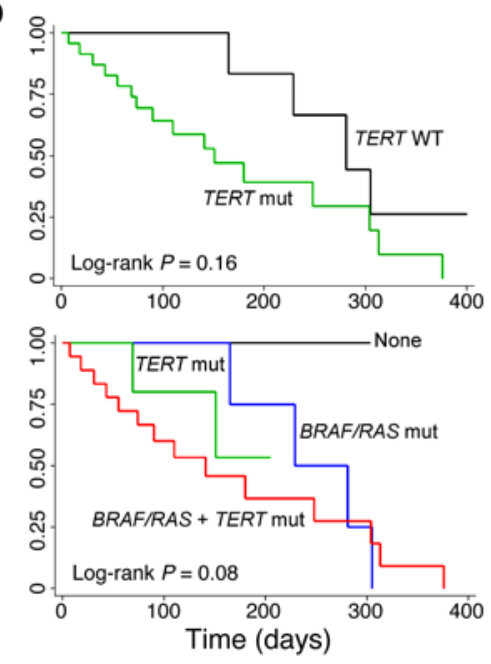

Figure 3. TERT promoter mutations in thyroid cancers. (A) Location and overall frequency of TERT promoter mutations in PDTCs and ATCs. (B) Oncoprints of TERT promoter mutations vs. BRAF and RAS in (top) PTCs from TCGA ( $n=381$ ); (middle) PDTCs $(n=84)$; and (bottom) ATCs ( $n=33)$. (C) Allelic frequency of TERT promoter mutations in thyroid cancers. Graph shows TERT mutant allelic frequency (MAF) corrected for tumor purity, determined based on allelic fraction of driver mutations (BRAF or RAS) for all three tumor types. (D) Kaplan-Meier survival in ATCs with log-rank $P$ values. Top: TERT-mutant vs WT. Bottom: WT, TERT-mutant with or without BRAF/RAS mutations. See also Supplemental Table 7.

mutations (mutated in at least 2 ATCs or 3 PDTCs) in DIS3, FAT1, POLE, RBM10, RAD54L, RECQL4, and SF3B1.

Mutations of genes encoding members of the WNT signaling pathway - i.e., CTNNB1 ( $\beta$ catenin), AXIN1, and APC - have previously been reported as genetic hallmarks of ATCs $(12,13)$, with mutation frequencies $>60 \%$ for CTNNB1 (reviewed in Supplemental Table 8). The deep-sequencing results in our cohort do not replicate these findings. We found a single tumor carrying a missense mutation (p.L347P) in CTNNB1, which is distinct from the CTNNB1-exon 3 gain-of-function hotspot previously reported in this disease. Variants in AXIN1 were found in 2/117 tumors, both without paired normals available and both of which may have represented low-frequency germline polymorphisms. A single truncating mutation in APC (p.Q1529X) was found in an ATC with an unusually high mutation burden. In addition, our results do not support a relevant role for mutations in genes in the apoptosis, Hedgehog, homologous recombination, immune response, insulin-like, JAK-STAT, tricarboxylic acid, nucleotide excision repair, polycomb, ubiquitination, or TGF $\beta$ pathways in PDTC and ATC tumorigenesis. With respect to mutations reported in single cases in a recent exome sequencing study of ATCs (21), we replicated these findings in the cyclin-dependent kinase inhibitors $C D K N 1 B$ and $C D K N 2 C$ (and found an additional truncating mutation in $C D K N 2 A$ ), as well as in other genes such as ERBB2, PTCH1, and DAXX (Supplemental Figure 1), but not in TRAF7 and NCOR1.

\section{Gene fusions}

Chromosomal rearrangements involving genes known to be translocated in thyroid tumors were frequent events in PDTCs (14\%) but were absent in ATCs and did not overlap with BRAF, RAS, TSHR, or STK11 mutations (Figure 1D). RET/PTC rearrangements were detected in 5 PDTCs and involved the most common RET partners CCDC6 and NCOA4. Translocations leading to PAX8-PPARG fusions were observed in 3 PDTCs, whereas fusions involving $A L K$ gene were detected in another 3 tumors. The kinase domain of ALK was recombined with three different upstream partners, including the known $S T R N$ and $E M L 4$, as well as CCDC149, a novel $A L K$ fusion partner, which is a coiled-coil family gene located on chromosome 4 (fusion included CCDC149 exons 1-10 and $A L K$ exons 20-29). The 11 PDTCs harboring gene fusions occurred in younger patients ( 49 vs. 58 years, $P=0.04$; Supplemental Table 5).

A single ATC without known driver mutations carried a $\mathrm{t}(15 ; 19)$ (q13;p13.1) translocation involving the NUT gene (NUTM1, NUT midline carcinoma, family member 1) and BRD4 (bromo- 
Table 3. Contingency analysis of TERT-BRAF/RAS mutations in thyroid cancers

\begin{tabular}{lcccccc} 
& \multicolumn{3}{c}{ PTC-TCGA $^{A}$} & \multicolumn{3}{c}{ PDTC + ATC $^{\mathrm{B}}$} \\
& TERT WT & TERT mutant & Total & TERT WT & TERT mutant & Total \\
BRAF/RAS WT & 103 & 4 & 107 & 29 & 13 & 42 \\
BRAF/RAS mutant & 243 & 31 & 274 & 30 & 45 & 75 \\
Total & 346 & 35 & 381 & 59 & 58 & 117
\end{tabular}

APapillary thyroid cancers from the TCGA study (1), $n=381$. ${ }^{\mathrm{P} P D T C}$ and ATC from the current study $(n=84+33)$. TERT and BRAF/RAS mutations significantly co-occur both in PTCs (contingency test: $\mathrm{OR}=3.3 ; P=0.03$ ) and in PDTCs and ATCs combined (contingency test: $\mathrm{OR}=3.4 ; P=0.004)$. Odds ratios and $P$ values are derived from 2-tailed Fisher's exact tests.

domain containing 4), resulting in an in-frame NUT-BRD4 fusion (NUT exons 1-2 and BRD4 exons 14-20). It was detected in an ATC with areas of PDTC in a 34-year-old woman who underwent total thyroidectomy and laryngopharyngectomy, plus radiotherapy, and who is alive 10 years after diagnosis. It clearly represents an outlier from the clinical behavior standpoint, which matches with her unique genetic alteration, involved in large-scale chromatin remodeling (34).

\section{Somatic CNAs}

Tumor purity of PDTCs was similar to PTCs (median tumor content $74 \%$ and $72 \%$, respectively) whereas it was much lower in ATCs (42\%) (Supplemental Figure 2). By correcting for tumor purity, we greatly enhanced our sensitivity for detecting CNAs even in most of the heavily infiltrated ATCs, as well as in some PDTCs. The ability of IMPACT to call arm-level CNAs was explored in 37 tumors that were simultaneously profiled by array-CGH. As seen in Supplemental Figure 3, CNA calls were efficiently replicated in both platforms. In addition, IMPACT identified CNAs that were not detected by array-CGH, particularly in heavily infiltrated tumors.

Whereas the genome of PTCs is largely diploid, CNAs in advanced thyroid tumors were common and widespread (Supplemental Figure 4). Interestingly, CNAs were more frequent in the ATCs and PDTCs that lacked driver mutations (Figure 5A). A similar subset of PTCs from the TCGA analysis also possessed a high prevalence of CNAs in the absence of driver mutations (1). Overall, 8 arm-level recurrent CNAs (losses of 1p, 8p, 13q, 15q, 17p, 22q, and gains of 1q and 20q; Figure 5A) were present at higher prevalence, an effect that persisted at a more conservative copy number threshold (see Methods).

Most of the CNAs occurred in a tumor type- and gene context-specific fashion (Figure $5 \mathrm{~B})$. Loss of $1 \mathrm{p}$ was marginally more frequent in PDTCs $(P=0.06)$, whereas $8 p$ and $17 p$ losses and $20 q$ gains were far more frequent in ATC genomes $\left(P<2 \times 10^{-4}\right.$ for all three). Chromosome 1p, 13q, and 15q losses were enriched in PDTCs without known driver mutations $\left(\chi^{2} P=0.03,2 \times 10^{-3}\right.$, and $3 \times 10^{-4}$, respectively), whereas loss of $22 \mathrm{q}$ was strongly associated with $R A S$-mutated PDTCs as compared with $B R A F$ tumors $\left(P=1 \times 10^{-3}\right)$ (Figure 5A).

Three out of 8 of the recurrent CNAs in the genomes of PDTCs and ATCs were associated with outcome. Patients with PDTCs with gains in chromosome $1 \mathrm{q}$ had worse survival $(\log -\operatorname{rank} P=0.06)$ (Figure 5C). In ATCs, 13q losses and 20q gains were associated with shorter survival (log-rank $P=0.02$ and 0.06 , respectively).

\section{Gene expression profiling: signaling and differentiation}

We used a subset of fresh-frozen specimens of 17 PDTCs and 20 ATCs to derive insights into the gene expression profiles of advanced thyroid cancers. The 37-tumor dataset was representative of the main driver genetic alterations described and included $13 B R A F, 12 R A S, 5$ with alterations in other drivers (NF1, NCOA4RET, CCDC149-ALK, NUT-BRD4, and STK11), and 7 without known driver mutations (Supplemental Table 9).

A principal component analysis efficiently separated both entities based on their global gene expression (Figure 6A). Only 2 tumors clustered out of their tumor type group: an ATC from a metastatic specimen (the single one in this subset of 20 ATCs) and a BRAF-mutant PDTC that was heavily infiltrated with macrophages (tumor purity $=46 \%$, high M2-macrophage signature score, see also Figure 7A).

MAPK signaling: the BRAF-RAS score. As a consequence of their genomic simplicity, the key oncogenic drivers of PTCs are associated with distinct biological, signaling, and gene expression properties. Pertaining to this study, the recently published TCGA analysis of 390 PTCs showed clear gene expression differences between BRAF and RAS tumors, which were used to construct a BRAF-RAS score (BRS) (1). We aimed to evaluate whether these driver-dependent gene expression characteristics persist in advanced cancers, which harbor a more complex cancer genome. Sixty-seven out of the 71 genes in the BRS were present in the mRNA array and were assessed in our PDTCs and ATCs with known driver mutation status. We found a high correlation between BRS values and BRAF/RAS mutation status (Figure 6, B and C). All $13 \mathrm{BRAF}^{\mathrm{V} 600 \mathrm{E}}$-mutated PDTCs and ATCs were BRAFlike. However, although RAS-mutant PDTCs were strongly RASlike, RAS-mutant ATCs were BRAF-like $\left(P=3 \times 10^{-3}\right)$, suggesting that a high MAPK transcriptional output is a characteristic property of ATCs, regardless of the driver mutation (Figure 6C and Supplemental Figure 5).

Macrophage infiltration. ATCs are known to be extensively infiltrated with macrophages $(22,23)$. Although it is assumed that these are M2 macrophages, which promote tumorigenesis, this has not been proven. We applied a previously characterized signature (35) of 78 genes overexpressed in M2 macrophages (68 of which were represented in our array) to the 37 tumors (Figure $7 \mathrm{~A}$ and Supplemental Figure 6) and found that it was sufficient to discriminate ATCs from the great majority of PDTCs, which are less prone to macrophage infiltration. The only 3 PDTCs that clustered with ATCs had a lower median estimated tumor purity than the 
A

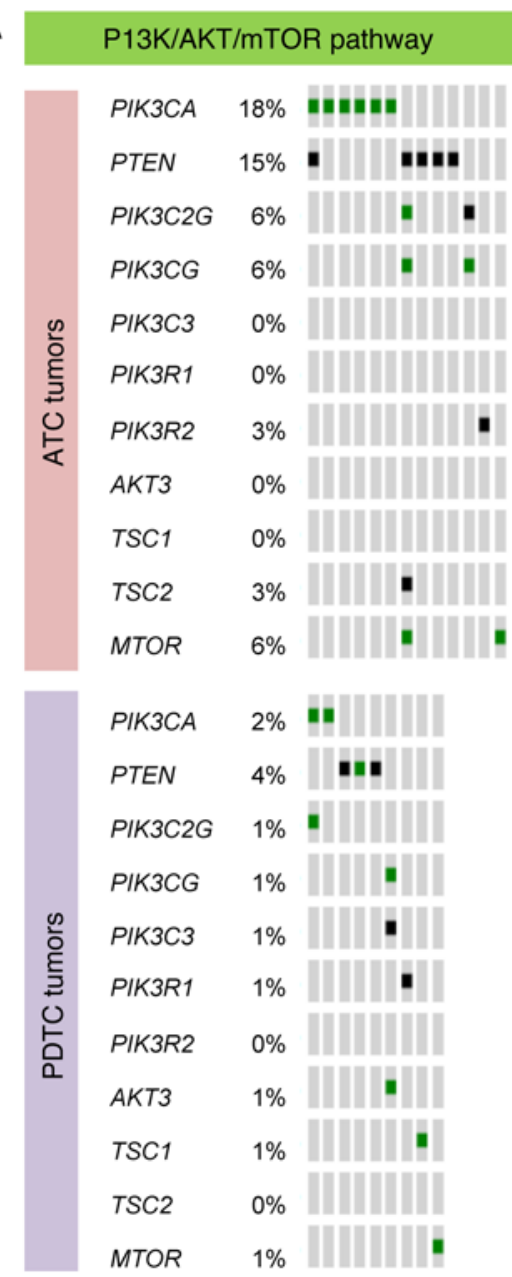

B

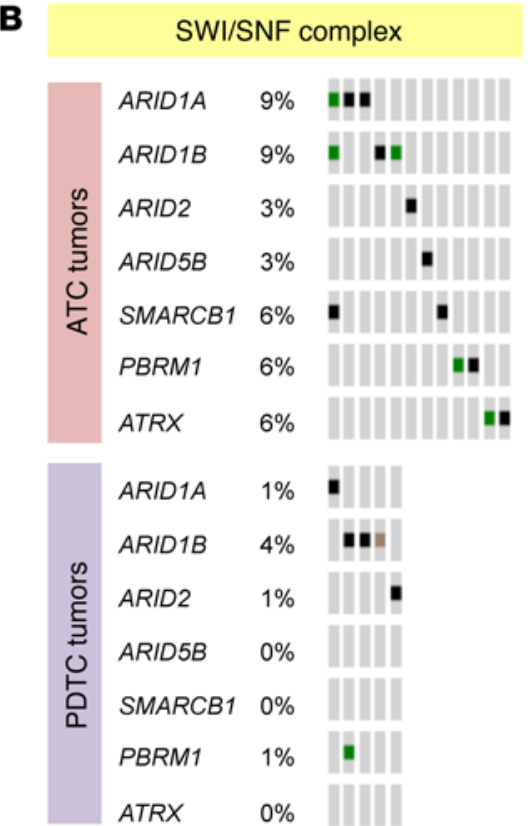

$\mathbf{E}$

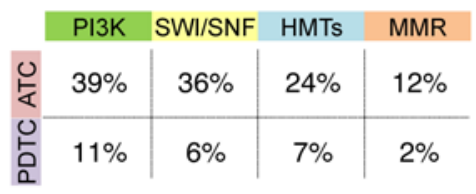

Genetic alteration

missense $\mathbf{\text { truncating }}$ inframe
C

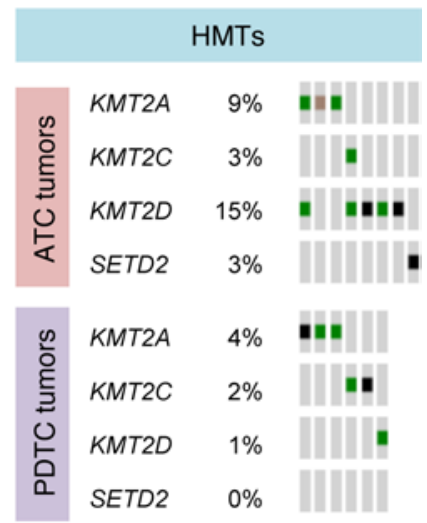

D
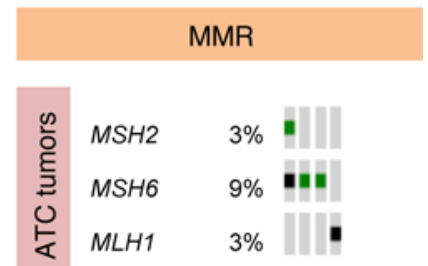

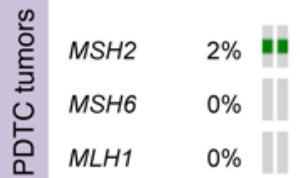

Figure 4. Pathways and novel functional groups mutated in advanced thyroid tumors. Expanded oncoprints of genes belonging to the indicated functional categories, as defined in Figure 1C. Samples are divided by tumor type (ATC or PDTC) within each panel. Only altered cases, out of 117 tumors, are shown. Missense, truncating, and in-frame mutations are represented as green, black, and brown squares, respectively. (A) PI3K/AKT/mTOR pathway (includes PIK3CA, PTEN, PIK3C2G, PIK3CG, PIK3C3, PIK3R1, PIK3R2, AKT3, TSC1, TSC2, and MTOR); (B) SWI/SNF chromatin remodeling complex (ARID1A, ARID1B, ARID2, ARID5B, SMARCB1, PBRM1, and ATRX); (C) HMTs (KMT2A, KMT2C, KMT2D, and SETD2); and (D) MMR (MSH2, MSH6, and MLH1). (E) Percentage of tumors altered for each functional category and tumor type.

rest of the PDTCs, suggestive of significant stromal contamination (46\% vs. $84 \%$, respectively; median purity in ATCs was $36 \%)$. Consistent with this, the prevalence of likely clonal mutations in ATCs, such as BRAF and TERT, is higher in this study than that described in prior reports (refs. 10, 17-20, 36-38, and Supplemental Table 8), probably due to IMPACT's deeper coverage.

Thyroid differentiation score. Loss of expression of thyroid differentiation markers is one of the hallmarks of advanced thyroid cancers and has profound consequences for the clinical management of these patients, who are usually refractory to radioiodine therapy due to loss of expression of NIS (sodium iodide symporter, SLC5A5) and other genes required for iodine incorporation. The TCGA analysis of PTCs used a thyroid differentiation score (TDS) consisting of 16 genes involved in iodine metabolism and thyroid specification to investigate driver-dependent effects on these parameters. We compared the TDS in PDTCs and ATCs with 8 PTCs profiled with the same platform (39). Overall, PDTCs and PTCs did not differ greatly, whereas ATCs had profoundly suppressed mRNA levels for TG, TSHR, TPO, PAX8, SLC26A4, DIO1, and DUOX2 genes (Figure
7B and Supplemental Table 9). None of the tumor types expressed THRB, DUOX1, SLC5A5, or SLC5A8. Unsupervised clustering based on the TDS discriminated ATCs from PDTCs (Supplemental Figure 7), with the exception of 3 PDTCs. These clustered with ATCs and corresponded to patients who died of the disease, two of whom were the only BRAF ${ }^{\mathrm{V} 600 \mathrm{E}}$-mutated PDTCs in this subset.

Finally, we evaluated the relationship between TDS, BRS, and the driver mutations of these tumors. As shown in Figure 7C, TDS and BRS were correlated in PDTCs, as tumors with low TDS corresponded with BRAF-like specimens, whereas this correlation was completely lost in ATCs. Similarly, BRAF-mutated PDTCs showed a marked decrease in TDS when compared with their RAS-mutated counterparts, whereas ATCs were homogeneously undifferentiated (Figure 7D).

\section{Discussion}

Most PDTCs and ATCs are thought to arise from preexisting PTCs based on their frequent co-occurrence in the same tumor specimen, where they consistently share a driver mutation $(36,40)$. 

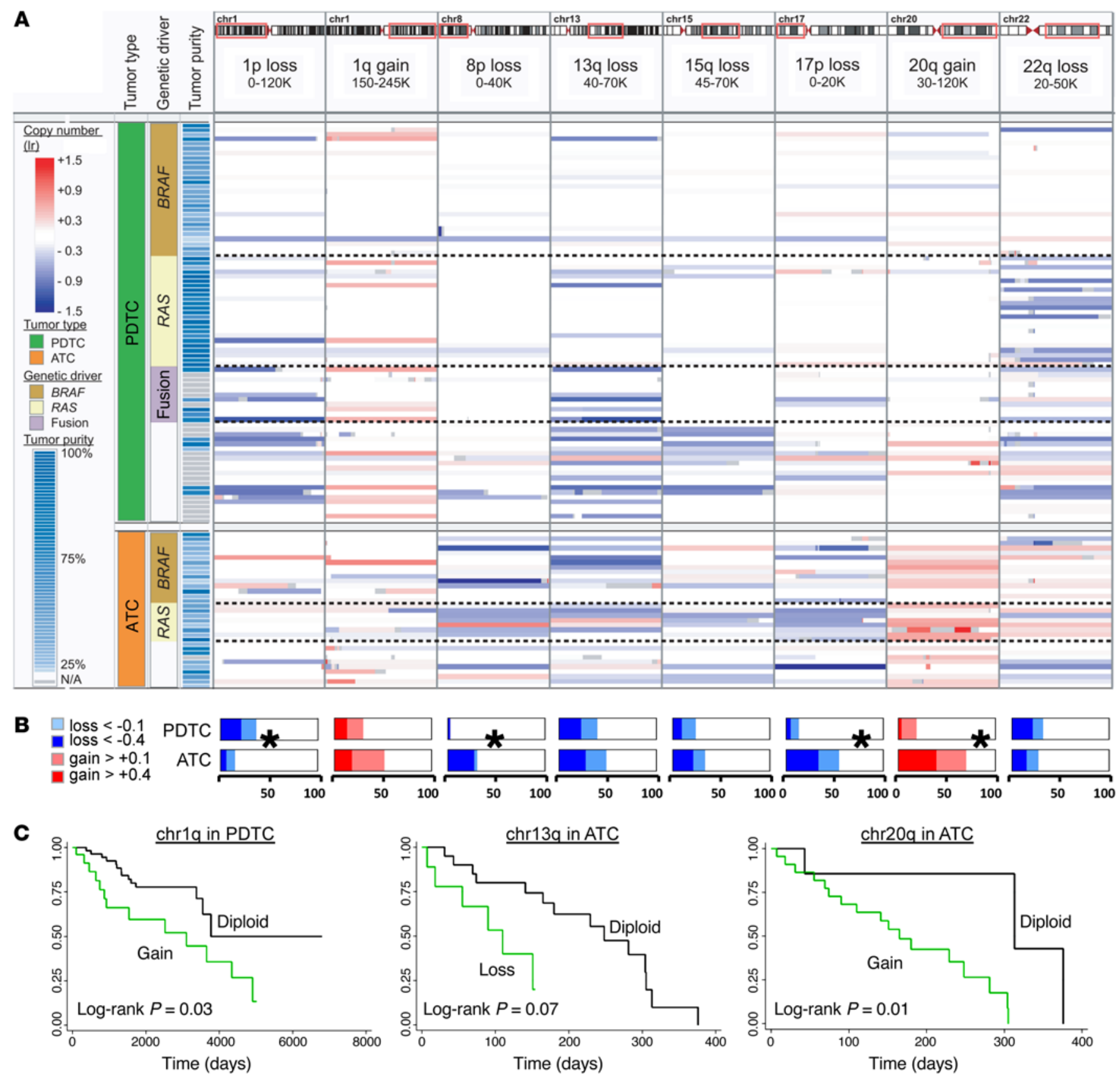

Figure 5. Recurrent MSK-IMPACT-derived CNAs found in 84 PDTC and 33 ATC. Representation of arm-level regions recurrently gained or lost in PDTCs and/or ATCs. CNAs were corrected for tumor purity in each sample with known driver mutations (see Methods and Supplemental Figure 2). (A) IGV representation of the altered chromosomal regions, with approximate locations shown on the top panel (genome build hg19), expressed as red (gain) or blue (loss), with shading intensity proportional to the log-ratio (Ir) values. Samples are grouped by tumor type and sorted by genetic driver alteration: BRAF, RAS, fusions (RET/PTC, PAX8-PPARG, and ALK), or none/unknown. Color key and annotations are shown on the left. (B) Frequencies of the indicated CNAs in PDTCs and ATCs. Copy number gains (red) or losses (blue) were defined using two Ir thresholds: \pm 0.1 (lighter shading) and \pm 0.4 (darker shading). Asterisks denote significant differences expressed as Fisher's exact test $P$ values for \pm 0.4 threshold: PDTC, 0.06 for $1 p$ loss; ATC, $<2 \times 10^{-4}$ for $8 p$ loss, $17 p$ loss, and 20q gain. (C) Kaplan-Meier survival curves for PDTCs harboring chromosome $1 q$ gain (left, log-rank $P$ values for \pm 0.1 and \pm 0.4 thresholds are 0.03 and 0.06 , respectively) and for ATCs with $13 q$ loss (middle, $P=0.07$ and 0.02 ) or $20 q$ gain (right, $P=0.01$ and 0.06 ).

Our results, analyzed in the context of the PTC TCGA study, provide insights into tumor microevolution and lend support to this model of tumorigenesis. Particularly compelling in this regard is the fact that mutations in the TERT promoter, which are known to activate its transcription, display a stepwise increase in frequency along the spectrum of disease progression (9\% in PTCs, $40 \%$ in
PDTCs, and 73\% in ATCs) (1, 17-20). Interestingly, TERT mutations are subclonal in the few PTCs that harbor them, whereas they are clonal in PDTCs and ATCs, pointing to selection during tumor evolution, possibly by inducing cell immortalization. TERT promoter mutations also track with virulence of advanced disease. They are significantly associated with $B R A F$ or RAS mutations, 
A

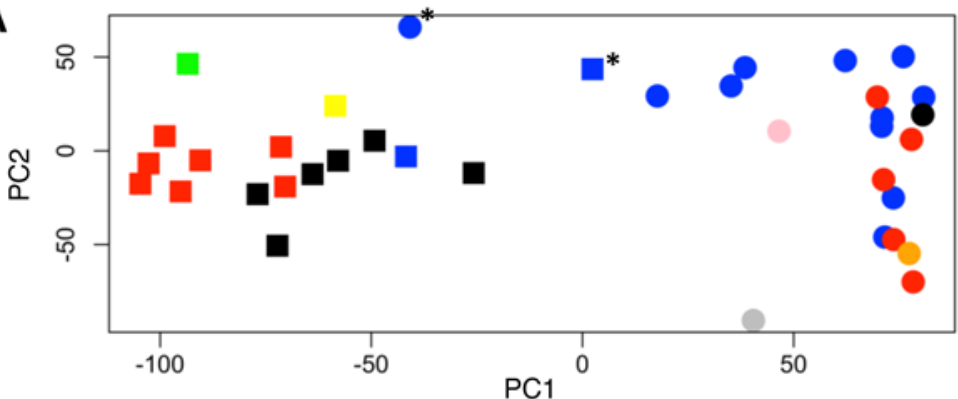

Tumor type: $\square$ PDTC

O ATC

Driver alteration ${ }^{3}$

B

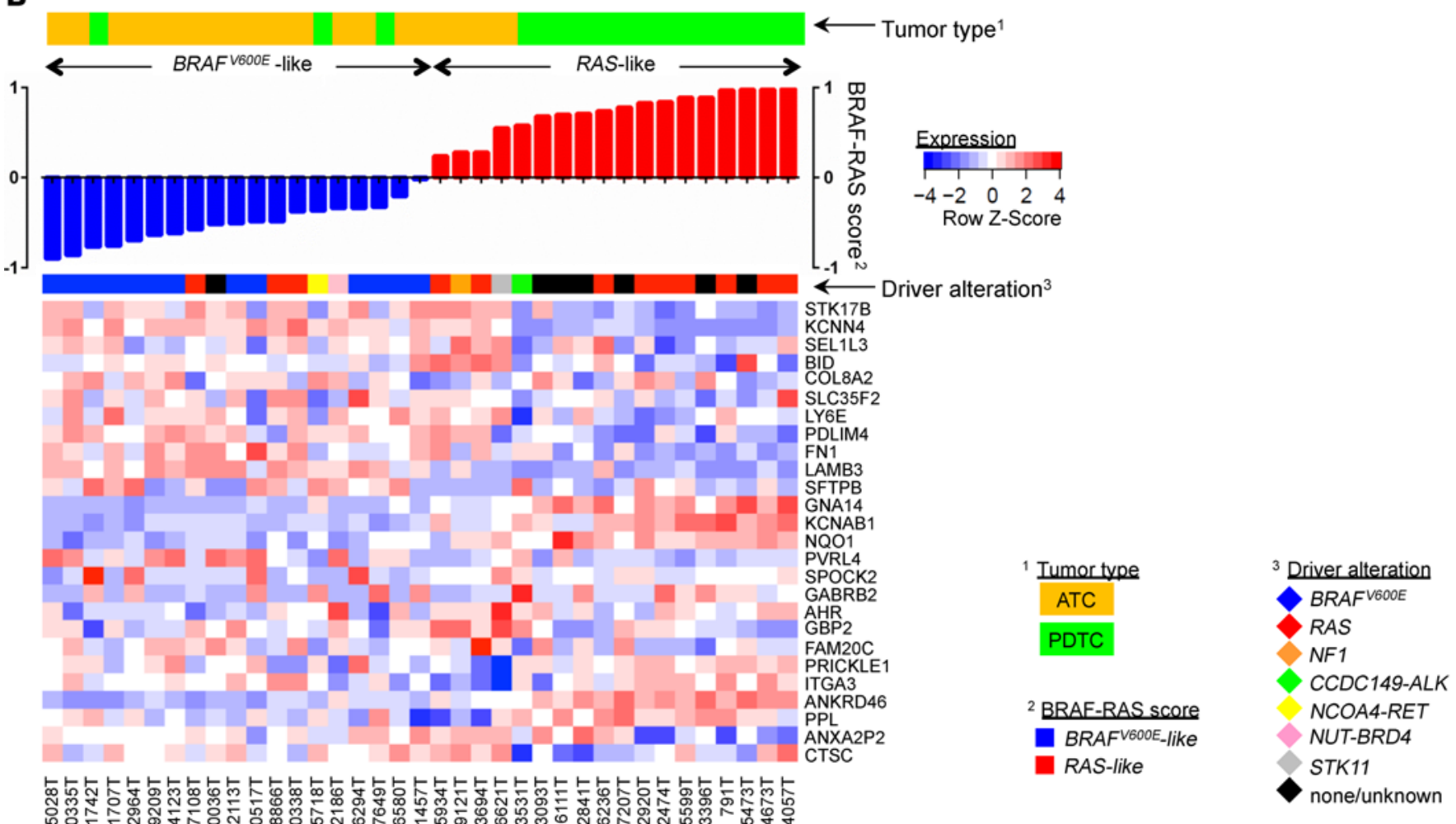

C

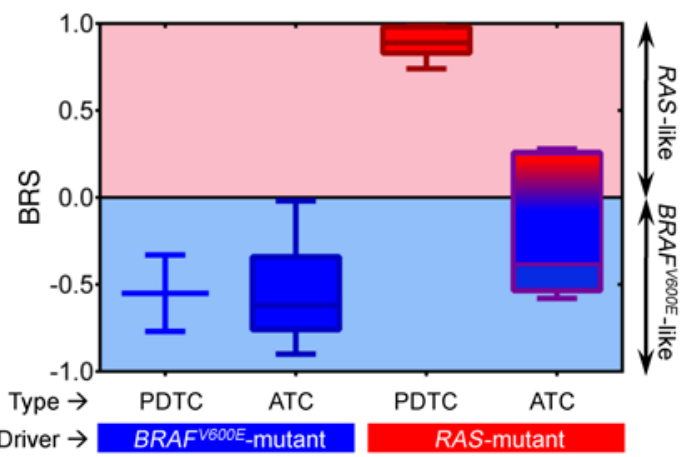

consistent with the proposed mechanism by which mutations in the promoter, by generating novel consensus motifs for the ETS family of transcription factors, promote TERT overexpression in cells with constitutive activation of MAPK signaling.
Figure 6. Principal component analysis (PCA) and BRS of 17 PDTCs and 20 ATCs. (A) Two-dimensional PCA discriminates PDTCs (squares) from ATCs (circles). Color-coding for driver alterations is shown in B. Asterisks represent ATC and PDTC outliers; see text. (B) Heatmap generated by applying the 67-gene BRS signature to advanced thyroid tumors. Expression values are displayed as Z-scores after scaling the values of each gene across the 37 samples. The 26 most informative genes are shown; the complete 67-gene signature is shown in Supplemental Figure 5. Samples are sorted by ascending BRS score: (BRAF ${ }^{V 600 E}$-like on the left and $R A S$-like on the right) and annotated for tumor type and driver alteration. (C) Detailed comparison of driver mutation vs. BRS in BRAFand RAS-mutant PDTCs and ATCs. Paradoxically, RAS-mutant ATCs are primarily $B R A F^{V 600 E}$-like (Mann-Whitney $U$ test, $P=0.003$ ). Box plots were generated using the Tukey method: horizontal lines within each box represent median values, box heights symbolize the IQR (IQR = Q3-Q1); Q3 and Q1 quartiles correspond to the top and bottom boundaries of the box, respectively; whiskers represent values up to 1.5 times IQR greater than Q3 (top: Q3 + $1.5 \times$ IQR) or smaller than Q1 (bottom: Q1 - $1.5 \times$ IQR).

By using a platform with a depth of sequencing optimized to identify mutations in tumors known to be associated with abundant stromal contamination, primarily by tumor associated macrophages (TAMs) $(22,23)$, we identified key genetic lesions that 

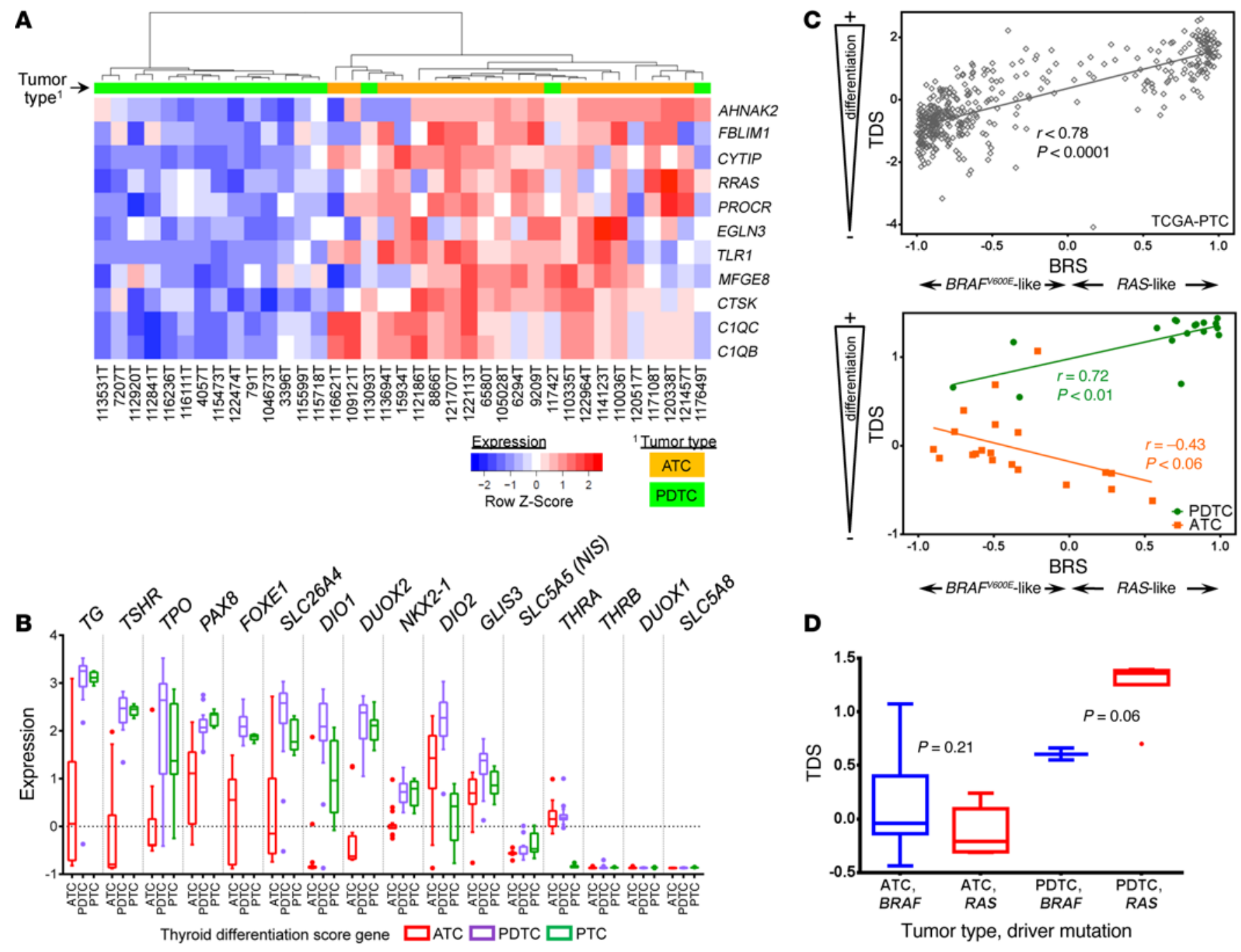

D

Figure 7. M2 macrophage signature and TDS of 17 PDTCs and 20 ATCs. (A) Unsupervised clustering based on a 68-gene M2-macrophage signature in advanced thyroid tumors (35). Expression values are displayed as Z-scores after scaling the values of each gene across the 37 samples. The 11 most discriminatory genes (variance greater than 2) are shown; the complete 68-gene signature is shown in Supplemental Figure 6. ATCs clearly cluster apart from PDTCs consistent with their extensive macrophage infiltration. (B) Relative expression of the 16 genes of the TDS in 20 ATCs and 17 PDTCs, compared with 9 PTCs from He et al. (39) evaluated with the same mRNA array platform. ATCs have low TDS values for virtually all TDS genes, whereas PDTCs are comparable to PTCs. The 16-gene TDS signature discriminates ATCs and PDTCs (see unsupervised clustering in Supplemental Figure 7). (C) Correlation plots between TDS and BRS in PTCs from TCCA (top) and PDTCs and ATCs (bottom). Trend lines, Pearson's correlation coefficients ( $r$ ) and associated $P$ values are shown in the graphs. TDS and BRS are positively correlated in PTCs $(r=0.74, P<0.0001)$ and PDTCs $(r=0.72, P<0.01)$; i.e., RAS-like tumors tend to be more differentiated than BRAF-like cancers. This relationship is lost in ATCs, which are profoundly undifferentiated $(r=-0.43, P=0.06)$. (D) Comparison of TDS values in BRAF- and RAS-mutated PDTCs and ATCs. Whereas ATCs are undifferentiated regardless of their driver alteration (Mann-Whitney $U$ test, $P=0.21), B R A F$-mutated PDTCs show a decrease in TDS compared with their $R A S$-mutant counterparts $(P=0.06)$. Box plots from $\mathbf{B}$ and $\mathbf{D}$ were generated using the Tukey method: horizontal lines within each box represent median values; box heights symbolize the IQR (IQR = Q3-Q1); Q3 and Q1 quartiles correspond to the top and bottom boundaries of the box, respectively; and whiskers represent values up to 1.5 times IQR greater than Q3 (top: Q3 + $1.5 \times$ IQR) or smaller than Q1 (bottom: Q1-1.5 × IQR). Values outside these limits are considered outliers and are represented by dots.

distinguish PDTCs from ATCs (i.e., TP53, TERT, and genes encoding effectors in the PI3K pathway). This includes genetic defects that implicate functional programs not previously associated with thyroid cancer, such as the SWI/SNF complex, HMTs, and others.

With respect to the main driver alterations, BRAF mutations were less prevalent in advanced tumors compared with PTCs, whereas RAS mutations were more frequent. Rearrangements commonly seen in radiation-induced and, to a lesser extent, in sporadic PTCs (RET/PTC, PAX8-PPARG and ALK fusions) were present in a subset of PDTCs but absent in the ATCs we sampled
(9, 41, 42). NF1 mutations were only found in ATCs in our series. The TCGA study of PTCs showed that BRAF- and RAS-mutant tumors exhibited profound differences in their clinical and histological characteristics and in their gene expression profile. The BRS is a 71-gene panel that distinguishes $B R A F^{V 600 E}$ from $R A S$-mutant PTCs. It was highly correlated to the transcriptional output of the MAPK pathway, which was highest in BRAF-mutant cancers. This is explainable because ERK activation in RAS-mutant cells induces a negative feedback that disrupts RAF dimerization, thus attenuating pathway output. By contrast, BRAF ${ }^{\mathrm{V} 600 \mathrm{E}}$ signals as 
a monomer and is unresponsive to this constraint, resulting in a greater flux through the pathway $(1,43)$. We found that these sharp demarcations between $B R A F$ - and RAS-mutant disease persisted in PDTC but were largely lost in ATC. PDTCs that met the standard histological definition of that entity (Turin proposal, ref. 26) were strongly associated with RAS mutations. By contrast, those defined based on the presence of high mitotic rate and necrosis irrespective of other characteristics (27) were markedly enriched for $B R A F$. They also had distinct clinical behaviors: BRAF-mutant PDTCs primarily developed locoregional nodal metastases, whereas RAS-mutant PDTCs presented with distant metastases. The BRS tracked with the underlying driver mutation in PDTCs but not in ATCs. This was also true for a score derived from a gene set consisting of mRNAs encoding proteins required for the differentiated function of thyrocytes (the TDS). The greater genomic complexity of ATCs may account for blurring the association of gene expression with the nature of the underlying driver mutation, in particular because of the higher frequency of mutations of genes encoding chromatin modifiers or genes that activate parallel pathways. Interestingly, even ATCs with $R A S$ or other mutations tend to be BRAF-like, as defined by the BRS. In addition, ATCs are extensively infiltrated by TAMs. Accordingly, nonhierarchical analysis of a gene set that defines M2 macrophages clearly separated ATCs from PDTCs. It may be that the greater cellular heterogeneity of ATCs may account in part for the attenuation of the oncogenic driver effects on gene expression.

EIF1AX, which encodes for a key component of the translation preinitiation complex (PIC), is mutated in only $1 \%$ of PTCs but in approximately $10 \%$ of PDTCs and ATCs. The concordance of EIF1AX with RAS mutations is extremely strong, which is distinct from PTC, where these are largely mutually exclusive. The biological consequences of this association are currently unknown. The mutations of EIF1AX cluster in specific N- and C-terminal residues. The C-terminal p.A113 splice mutation is specific to thyroid cancer and predicts for alternative usage of a cryptic splice acceptor within exon 6, resulting in a 12-amino acid in-frame deletion. Our analysis of RNASeq data from two cases with this mutation in the PTC TCGA confirms this prediction (not shown). EIF1A plays a key role in regulating the conformation of the PIC and in scanning for the AUG initiation codon, which is disrupted in distinct ways by N-terminal and C-terminal mutations in yeast (44). Interestingly, EIF1AX mutations are mutually exclusive with alterations in any of the PI3K/AKT/mTOR pathway members, suggesting that they may confer overlapping functional gains. In addition, EIF1AX mutations are predictive of worse survival in PDTCs, providing a potentially useful marker for risk stratification in a heterogeneous disease in need of better prognostic indicators $(45,46)$.

Both entities differed in overall mutation burden, which was significantly higher in ATCs. Within PDTCs, a higher number of mutations was associated with larger tumors, presence of metastasis, and shorter survival. TP53 mutations, in particular, distinguished ATCs from PDTCs (73\% vs. $8 \%$, respectively). CNAs, some of which had been previously reported at very low frequencies (47-51), proved to be distinctive of each tumor type. PDTCs have a greater frequency of $1 p$ losses, whereas $8 p$ and $17 p$ losses, as well as $20 q$ gains, were more common in ATCs. Interestingly, 22q losses were strongly associated with $R A S$-mutant PDTCs. Loss of the 22q tumor suppressor gene NF2, which encodes for merlin, has been recently implicated in this association. Consistent with this, the combined activation of oncogenic Ras with Nf2 loss leads to development of PDTC in mice. This is because inactivation of the Hippo pathway through merlin loss leads to a YAP-TEAD-dependent transcriptional activation of oncogenic and WT RAS, thus enhancing MAPK transcriptional output and promoting transformation $(1,52)$.

We compared the results of our targeted cancer gene NGS (IMPACT) approach with a recently reported whole exome sequencing (WES) study of 22 ATCs (21). The greater depth of sequencing achieved by IMPACT $(739 \times$ vs. $264 \times$ in the WES study) may explain the differences observed between both platforms in the frequency of TP53 (73\% by IMPACT vs. $27 \%$ by WES), BRAF (45\% vs. $27 \%$ ), PIK3CA (18\% vs. $9 \%$ ), and PTEN (15\% vs. $0 \%$ ) and may improve detection of subclonal events. This is particularly relevant in ATCs because of their low tumor purity, which calls into question the suitability of WES as the platform of choice. Moreover, the WES approach failed to detect mutations in members of the SWI/SNF and HMT functional groups that we report here and that likely play a fundamental role in the biology of these tumors. We acknowledge, however, the drawbacks of sequencing a limited set of cancer genes. For instance, RASAL1, USH2A, HECTD1, $M L H 3$, and $M S H 5$, which were rarely mutated in ATCs by WES, were not included in IMPACT. Two of these, $M L H 3$ and MSH5, are MMR genes, a functional group that we find to be disrupted in at least $12 \%$ of ATCs.

The biological consequences of the novel drivers (e.g., EIF1AX and RAS) and functional groups (SWI/SNF) in the context of thyroid tumorigenesis remain to be explored. Others recapitulate phenotypes observed in genetically engineered mouse models of advanced thyroid cancers, such as PTEN and TP53 (53), BRAF and TP53 (54), RAS and TP53 (55), RAS and NF2 (52), and $B R A F$ and PIK3CA (56).

The findings reported here provide tools that can be leveraged to improve the molecular diagnosis of these clinical entities, many of which likely have prognostic implications. Particularly relevant is the strong association of PDTC-Turin tumors with RAS mutations, whereas PDTC-MSK tumors were strongly associated with $B R A F$. In addition, $R A S$ - and BRAF-mutant PDTCs have distinct tropism for metastases, with the former tending to home at a distance, whereas the latter metastasize to locoregional lymph nodes. Moreover, the discovery in well-differentiated tumors of subclonal mutations of genes that we show to be enriched in advanced disease should raise particular concerns. This also opens a path to explore the biology of novel genetic associations that may point to tumor dependencies that can be exploited therapeutically.

\section{Methods}

Patient tissue samples. PDTC and ATC samples were randomly selected from the pathology department files of the institution from 1986-2015. ATCs were classified according to the last WHO classification of endocrine tumors, whereas PDTCs were defined as follows: (i) according to the Turin proposal, by architectural and high-grade features (mitosis and necrosis), the presence of a solid/ nested/insular growth, the absence of nuclear features of PTC, and either convoluted nuclei, mitotic activity $\geq 3 \times 10$ high power fields (HPF), or tumor necrosis (26); and (ii) as a carcinoma display- 
ing high mitotic activity ( $\geq 5$ mitosis/10 HPF, $\times 400$ ) and/or tumor necrosis, and showing follicular cell differentiation at the morphological or immunohistochemical level (27). MSK-IMPACT targeted sequencing was performed in all 117 tumors (84 PDTCs and 33 ATCs): 80 from formalin-fixed paraffin-embedded tissues (FFPE) and 37 from fresh-frozen material. The 37 frozen tumors, 17 PDTCs and 20 ATCs, were also expression profiled with Affymetrix U133 plus 2.0 array and with the Agilent SurePrint G3 CGH 1x1M arrayCGH platform to validate copy number calls.

Single nucleotide variant and indel calling and filtering. Single nucleotide variants (SNVs) and short indels ( $<30$ bp in length) were automatically annotated by the MSK-IMPACT pipeline, as previously described (24). Tumor samples without paired normals (11/117; 5 ATCs and 6 PDTCs) were compared against pooled normals. All variants were annotated based on the information available in catalog of somatic mutations in cancer (COSMIC; http://cancer.sanger.ac.uk/ cosmic), NCBI-dbSNP (http://www.ncbi.nlm.nih.gov/snp), and the 1,000 Genomes Project (http://www.1000genomes.org/). Variants highlighted in this study were subsequently manually reviewed. For the 106 tumors with paired normals, all variants confirmed as somatic were reported, regardless of location and clonality. For tumors compared against pooled normals, MSK-IMPACT automatically called SNVs with reported frequencies $<1 \%$. We manually reviewed the 11 tumors fulfilling these criteria as follows: (i) keeping variants reported in COSMIC; (ii) removing variants reported as polymorphic (with an reference sequence code in dbSNP); and (iii) removing variants with allele frequencies that were $>10 \%$ of the allelic fraction of the driver mutation in the same tumor.

MSK-IMPACT sequencing data is publicly available at the cBioPortal for Cancer Genomics (http://www.cbioportal.org/). Mutation plots were generated using the OncoPrinter (v1.0.1) and MutationMapper (v1.0.1) tools, which are available at the cBioPortal $(57,58)$.

Chromosomal rearrangements were called for genes whose introns were covered by MSK-IMPACT, which included most of the previously reported fusions in thyroid tumors (with the notable exceptions of NTRK1 and NTRK3).

CNAs and estimation of tumor purity. DNA CNAs were primarily called from IMPACT by comparing sequence reads of targeted regions in tumors relative to a standard diploid normal sample, as described (24). Although IMPACT targets a discrete number of exons in each chromosome arm, it efficiently identified arm-level chromosomal genetic gains and losses, as confirmed by a genomewide Agilent SurePrint G3 CGH 1x1M array-CGH platform in a subset of 37 advanced thyroid tumors (Supplemental Figure 3), which showed excellent agreement in the copy number calls between the two methodologies.

As the macrophage infiltration of advanced thyroid tumors (particularly ATCs) can impact the sensitivity of CNA detection, we corrected CNA values for each tumor based on tumor purity. Tumor purity was calculated based on the mutant allele frequencies of clonal heterozygous somatic mutations in regions lacking overt CNAs (see also Supplemental Figure 2). For tumors with a BRAF or RAS mutation (heterozygous mutations considered clonal events) (59), purity was calculated by doubling the frequency of the alternate allele (e.g., in a tumor with a $\mathrm{BRAF}^{\mathrm{V} 600 \mathrm{E}}$ mutation frequency of 0.45 , tumor purity was $90 \%$ ). For tumors without BRAF or RAS mutations, we used other gene mutation frequencies as purity esti- mators. In tumors lacking canonical drivers, we used an average of all likely clonal events. We discarded likely subclonal events (e.g., mutations with disproportionately low allelic frequencies compared with other somatic variants). We discarded tumor suppressors (i.e., TP53, NF1) as purity estimators due to possible coexisting loss-of-heterozygosity events. Chromosomal regions harboring the drivers (typically BRAF or RAS) were confirmed to be diploid before using those genes as purity estimators. Altogether, we derived tumor purity data from $75 B R A F / R A S$-mutated tumors and used alternative genes for 19 others. Purity could not be estimated with confidence for 23 tumors (22 PDTCs and 1 ATC).

Segmented genome-wide copy number was corrected for tumor purity in the following manner. For all segments where $r$ is the $\log _{2}$ ratio of segmented copy number, then $R=2^{r}$ and $\alpha$ is the estimated tumor purity, the copy ratio in cancer cells of a cellularly heterogeneous sample is therefore: $R^{\prime}=(R / a)-([1-a] / a)$ for all $r>\log _{2}(1-a)$, otherwise $R^{\prime}=(r / \min [r]) \min \left(R^{\prime}\right)$.

The final purity-corrected copy number is $\log _{2}\left(R^{\prime}\right)$ for all segments in the specimen. CNAs were determined using the purity-corrected segmented data utilizing one of two thresholds $( \pm 0.1$ and the more conservative \pm 0.4 threshold). All analyses were expressed at \pm 0.4 , with the exception of survival analyses. As focal events were few, the analyses described here focused on larger and overt arm-level CNAs. Given the challenge of definitively establishing thresholds of heterozygous loss and homozygous deletions in impure tumors (signal compression), our analysis excluded homozygous CNAs. CNAs were visualized in the Integrative Genomics Viewer (IGV), version 2.3.57 (https://www.broadinstitute.org/igv/) (60).

Gene expression. We performed mRNA expression on 37 tumors using the Affymetrix U133 plus 2.0 array. Expression normalization was performed using the gcRMA method (61). Statistical analyses were performed using the R statistical programming language (62). The lists of genes for the BRS and TDS scores were obtained from the TCGA study on PTC (1). Of the 71 BRS genes, 67 were present on our array and were used to compute the BRS score as described (1). We performed sample-wise scaling on the gene-expression profiles of the 9 PTC tumors, which were derived from a published study (39). The lists of differentially expressed genes between M1 and M2 macrophages (35) were from the MSigDB (63). The data discussed in this publication have been deposited in NCBI's Gene Expression Omnibus (GEO) (64) and are accessible through GEO Series accession number GSE76039.

Clinical associations. Statistical analyses were performed using STATA/IC (version 12; StataCorp LP). Pathological characteristics were only available for resected ATCs. PDTCs had complete clinical and pathological data. Survival was recorded as of April 2015. All associations reported in PDTCs remained significant when only considering PDTCs based on the Turin criteria definition.

Statistics. Distribution of mutation frequencies in PDTCs versus ATCs was assessed by Fisher's exact tests. Copy number distribution between multiple groups was evaluated with $\chi^{2}$ tests. Statistical analyses and graphic representations of mutation and CNA distribution were performed on GraphPad Prism 6.02 (GraphPad Software). Demographic and clinico-pathologic characteristics were compared using Pearson $\chi^{2}$ test for categorical variables and 2-tailed Student's $t$ test or Mann-Whitney $U$ test for continuous variables. Survival analyses were conducted with Cox proportional hazards models. Proportionality assumptions were tested with Schoenfeld residuals and log- 
log plots. Kaplan-Meier survival curves were built, and the log-rank test was used to assess for significance of the surviving function. A $P$ value less than 0.05 was considered significant.

Study approval. The study was approved by the Institutional Review Board of MSKCC.

\section{Author contributions}

IL, IG, and JAF designed the study; IL, TI, RS, JCRF, and GPK conducted experiments; TI, LB, RHS, NS, MFB, and RG acquired data; IL, LB, RS, JAK, RHS, MFB, CS, BST, IG, and JAF analyzed data; SD, BX, and RG provided samples or reagents; IL and JAF wrote the manuscript.

\section{Acknowledgments}

We thank Nancy Bouvier from the Center for Molecular Oncology for technical assistance.

Address correspondence to: James A. Fagin, Department of Medicine and Human Oncology and Pathogenesis Program, Memorial Sloan-Kettering Cancer Center, 1275 York Avenue, Box 296, New York, New York 10065, USA. Phone:646.888.2136; E-mail:faginj@ mskcc.org. Or to: Ian Ganly, Department of Surgery and Human Oncology and Pathogenesis Program, Memorial Sloan-Kettering Cancer Center, 1275 York Avenue, Box 296, New York, New York 10065, USA. Phone: 212.639.6244; E-mail: ganlyi@mskcc.org.
1. Cancer Genome Atlas Research Network. Integrated genomic characterization of papillary thyroid carcinoma. Cell. 2014;159(3):676-690.

2. Lawrence MS, et al. Mutational heterogeneity in cancer and the search for new cancer-associated genes. Nature. 2013;499(7457):214-218.

3. Smallridge RC, Marlow LA, Copland JA. Anaplastic thyroid cancer: molecular pathogenesis and emerging therapies. Endocr Relat Cancer. 2009;16(1):17-44.

4. Kojic SL, Strugnell SS, Wiseman SM. Anaplastic thyroid cancer: a comprehensive review of novel therapy. Expert Rev Anticancer Ther. 2011;11(3):387-402.

5. Smallridge RC, Copland JA. Anaplastic thyroid carcinoma: pathogenesis and emerging therapies. Clin Oncol (R Coll Radiol). 2010;22(6):486-497.

6. Wagle N, et al. Response and acquired resistance to everolimus in anaplastic thyroid cancer. $N$ Engl JMed. 2014;371(15):1426-1433.

7. Rosove MH, Peddi PF, Glaspy JA. BRAF V600E inhibition in anaplastic thyroid cancer. $N$ EnglJ Med. 2013;368(7):684-685.

8. Nikiforov YE, Nikiforova MN. Molecular genetics and diagnosis of thyroid cancer. Nat Rev Endocrinol. 2011;7(10):569-580.

9. Ricarte-Filho JC, et al. Mutational profile of advanced primary and metastatic radioactive iodine-refractory thyroid cancers reveals distinct pathogenetic roles for BRAF, PIK3CA, and AKT1. Cancer Res. 2009;69(11):4885-4893.

10. Garcia-Rostan G, et al. Mutation of the PIK3CA gene in anaplastic thyroid cancer. Cancer Res. 2005;65(22):10199-10207.

11. Pita JM, Figueiredo IF, Moura MM, Leite V, Cavaco BM. Cell cycle deregulation and TP53 and RAS mutations are major events in poorly differentiated and undifferentiated thyroid carcinomas. JClin Endocrinol Metab. 2014;99(3):E497-E507.

12. Garcia-Rostan G, Tallini G, Herrero A, D’Aquila TG, Carcangiu ML, Rimm DL. Frequent mutation and nuclear localization of beta-catenin in anaplastic thyroid carcinoma. Cancer Res. 1999;59(8):1811-1815.

13. Kurihara T, et al. Immunohistochemical and sequencing analyses of the Wnt signaling components in Japanese anaplastic thyroid cancers. Thyroid. 2004;14(12):1020-1029.

14. Fagin JA, Matsuo K, Karmakar A, Chen DL, Tang $\mathrm{SH}$, Koeffler HP. High prevalence of mutations of the p53 gene in poorly differentiated human thyroid carcinomas. J Clin Invest. 1993;91(1):179-184.
15. Donghi R, Longoni A, Pilotti S, Michieli P, Della Porta G, Pierotti MA. Gene p53 mutations are restricted to poorly differentiated and undifferentiated carcinomas of the thyroid gland. J Clin Invest. 1993;91(4):1753-1760.

16. Nakamura T, et al. p53 gene mutations associated with anaplastic transformation of human thyroid carcinomas. Jpn J Cancer Res. 1992;83(12):1293-1298.

17. Landa I, et al. Frequent somatic TERT promoter mutations in thyroid cancer: higher prevalence in advanced forms of the disease. JClin Endocrinol Metab. 2013;98(9):E1562-E1566.

18. Liu X, et al. Highly prevalent TERT promoter mutations in aggressive thyroid cancers. Endocr Relat Cancer. 2013;20(4):603-610.

19. Liu T, et al. The age- shorter telomere-dependent TERT promoter mutation in follicular thyroid cell-derived carcinomas. Oncogene. 2013;33(42):4978-4984

20. Melo M, et al. TERT promoter mutations are a major indicator of poor outcome in differentiated thyroid carcinomas. JClin Endocrinol Metab. 2014;99(5):E754-E765.

21. Kunstman JW, et al. Characterization of the mutational landscape of anaplastic thyroid cancer via whole-exome sequencing. Hum Mol Genet. 2015;24(8):2318-2329.

22. Caillou B, et al. Tumor-associated macrophages (TAMs) form an interconnected cellular supportive network in anaplastic thyroid carcinoma. PLoS One. 2011;6(7):e22567.

23. Ryder M, Ghossein RA, Ricarte-Filho JC, Knauf JA, Fagin JA. Increased density of tumor-associated macrophages is associated with decreased survival in advanced thyroid cancer. Endocr Relat Cancer. 2008;15(4):1069-1074.

24. Cheng DT, et al. Memorial Sloan Kettering-Integrated Mutation Profiling of Actionable Cancer Targets (MSK-IMPACT): A Hybridization Capture-Based Next-Generation Sequencing Clinical Assay for Solid Tumor Molecular Oncology. J Mol Diagn. 2015;17(3):251-264.

25. Kondo T, Ezzat S, Asa SL. Pathogenetic mechanisms in thyroid follicular-cell neoplasia. Nat Rev Cancer. 2006;6(4):292-306.

26. Volante M, et al. Poorly differentiated thyroid carcinoma: the Turin proposal for the use of uniform diagnostic criteria and an algorithmic diagnostic approach. Am J Surg Pathol. 2007;31(8):1256-1264.

27. Hiltzik D, et al. Poorly differentiated thyroid carcinomas defined on the basis of mitosis and necrosis: a clinicopathologic study of 58 patients. Cancer. 2006;106(6):1286-1295.

28. Martin M, et al. Exome sequencing identifies recurrent somatic mutations in EIF1AX and SF3B1 in uveal melanoma with disomy 3. Nat Genet. 2013;45(8):933-936.

29. Bell RJ, et al. Cancer. The transcription factor GABP selectively binds and activates the mutant TERT promoter in cancer. Science. 2015;348(6238):1036-1039.

30. Ito $\mathrm{T}$, et al. Unique association of $\mathrm{p} 53$ mutations with undifferentiated but not with differentiated carcinomas of the thyroid gland. Cancer Res. 1992;52(5):1369-1371.

31. Takeuchi Y, Daa T, Kashima K, Yokoyama S, Nakayama I, Noguchi S. Mutations of p53 in thyroid carcinoma with an insular component Thyroid. 1999;9(4):377-381.

32. Cremona CA, Behrens A. ATM signalling and cancer. Oncogene. 2014;33(26):3351-3360.

33. Helming KC, Wang X, Roberts CW. Vulnerabilities of mutant SWI/SNF complexes in cancer. Cancer Cell. 2014;26(3):309-317.

34. Alekseyenko AA, et al. The oncogenic BRD4NUT chromatin regulator drives aberrant transcription within large topological domains. Genes Dev. 2015;29(14):1507-1523.

35. Coates PJ, Rundle JK, Lorimore SA, Wright EG. Indirect macrophage responses to ionizing radiation: implications for genotypedependent bystander signaling. Cancer Res. 2008;68(2):450-456.

36. Nikiforova MN, et al. BRAF mutations in thyroid tumors are restricted to papillary carcinomas and anaplastic or poorly differentiated carcinomas arising from papillary carcinomas. J Clin Endocrinol Metab. 2003;88(11):5399-5404.

37. Liu Z, et al. Highly prevalent genetic alterations in receptor tyrosine kinases and phosphatidylinositol 3-kinase/akt and mitogen-activated protein kinase pathways in anaplastic and follicular thyroid cancers. J Clin Endocrinol Metab. 2008;93(8):3106-3116.

38. Soares P, et al. BRAF mutations and RET/ PTC rearrangements are alternative events in the etiopathogenesis of PTC. Oncogene. 2003;22(29):4578-4580.

39. He H, et al. The role of microRNA genes in papillary thyroid carcinoma. Proc Natl Acad Sci U S A. 2005;102(52):19075-19080.

40. Nikiforov YE. Genetic alterations involved in the transition from well-differentiated to poorly dif- 


\section{CLINICAL MEDICINE}

ferentiated and anaplastic thyroid carcinomas. Endocr Pathol. 2004;15(4):319-327.

41. Santoro M, et al. Ret oncogene activation in human thyroid neoplasms is restricted to the papillary cancer subtype. JClin Invest. 1992;89(5):1517-1522.

42. Santoro M, et al. RET activation and clinicopathologic features in poorly differentiated thyroid tumors. JClin Endocrinol Metab. 2002;87(1):370-379.

43. Pratilas CA, et al. (V600E)BRAF is associated with disabled feedback inhibition of RAF-MEK signaling and elevated transcriptional output of the pathway. Proc Natl Acad Sci U S A. 2009;106(11):4519-4524.

44. Fekete $\mathrm{CA}$, et al. $\mathrm{N}$ - and $\mathrm{C}$-terminal residues of eIF1A have opposing effects on the fidelity of start codon selection. ЕMBO J. 2007;26(6):1602-1614.

45. Sanders EM Jr, LiVolsi VA, Brierley J, Shin J, Randolph GW. An evidence-based review of poorly differentiated thyroid cancer. World J Surg. 2007;31(5):934-945.

46. Patel KN, Shaha AR. Poorly differentiated thyroid cancer. Curr Opin Otolaryngol Head Neck Surg. 2014;22(2):121-126.

47. Lee JJ, et al. Array-CGH identifies cyclin D1 and UBCH10 amplicons in anaplastic thyroid carcinoma. Endocr Relat Cancer. 2008;15(3):801-815.

48. Rodrigues RF, Roque L, Rosa-Santos J, Cid O, Soares J. Chromosomal imbalances associated with anaplastic transformation of follicular thyroid carcinomas. Br JCancer. 2004;90(2):492-496.
49. Wilkens $\mathrm{L}$, et al. Aberrations of chromosomes 5 and 8 as recurrent cytogenetic events in anaplastic carcinoma of the thyroid as detected by fluorescence in situ hybridisation and comparative genomic hybridisation. Virchows Arch. 2000;436(4):312-318.

50. Wreesmann VB, et al. Genome-wide appraisal of thyroid cancer progression. Am J Pathol. 2002;161(5):1549-1556.

51. Miura D, et al. Anaplastic thyroid cancer: cytogenetic patterns by comparative genomic hybridization. Thyroid. 2003;13(3):283-290.

52. Garcia-Rendueles ME, et al. NF2 loss promotes oncogenic RAS-induced thyroid cancers via YAP-dependent transactivation of RAS proteins sensitizes them to MEK inhibition. Cancer Discov. 2015;5(11):1178-1193.

53. Antico Arciuch VG, et al. Thyrocyte-specific inactivation of $\mathrm{p} 53$ and Pten results in anaplastic thyroid carcinomas faithfully recapitulating human tumors. Oncotarget. 2011;2(12):1109-1126.

54. McFadden DG, et al. p53 constrains progression to anaplastic thyroid carcinoma in a Braf-mutant mouse model of papillary thyroid cancer. Proc Natl Acad Sci U S A. 2014;111(16):E1600-E1609.

55. Champa D, Russo MA, Liao XH, Refetoff S, Ghossein RA, Di Cristofano A. Obatoclax overcomes resistance to cell death in aggressive thyroid carcinomas by countering Bcl2a1 and Mcl1 overexpression. Endocr Relat Cancer. 2014;21(5):755-767.

56. Charles RP, Silva J, Iezza G, Phillips WA, McMahon M. Activating BRAF and PIK3CA mutations
The Journal of Clinical Investigation

cooperate to promote anaplastic thyroid carcinogenesis. Mol Cancer Res. 2014;12(7):979-986.

57. Cerami E, et al. The cBio cancer genomics portal: an open platform for exploring multidimensional cancer genomics data. Cancer Discov. 2012;2(5):401-404.

58. Gao J, et al. Integrative analysis of complex cancer genomics and clinical profiles using the cBioPortal. Sci Signal. 2013;6(269):pl1.

59. Ghossein RA, Katabi N, Fagin JA. Immunohistochemical detection of mutated BRAF V600E supports the clonal origin of BRAFinduced thyroid cancers along the spectrum of disease progression. JClin Endocrinol Metab. 2013;98(8):E1414-E1421.

60. Robinson JT, et al. Integrative genomics viewer. Nat Biotechnol. 2011;29(1):24-26.

61. Wu Z, Irizarry RA, Gentleman R, MartinezMurillo F, Spencer F. A model-based background adjustment for oligonucleotide expression arrays. J Am Stat Assoc. 2004;99(468):909-917.

62. R Development Core Team. R: A Language and Environment for Statistical Computing. Vienna, Austria: R Foundation for Statistical Computing; 2015.

63. Subramanian A, et al. Gene set enrichment analysis: a knowledge-based approach for interpreting genome-wide expression profiles. Proc Natl Acad Sci U S A. 2005;102(43):15545-15550.

64. Edgar R, Domrachev M, Lash AE. Gene Expression Omnibus: NCBI gene expression and hybridization array data repository. Nucleic Acids Res. 2002;30(1):207-210. 